\title{
The Positivity of the Pressure in Thomas Fermi Theory $\star$
}

\author{
R. Benguria ${ }^{1 \star \star}$ and E. H. Lieb ${ }^{2}$
}

1 Department of Physics and

2 Departments of Mathematics and Physics, Princeton University, Princeton, New Jersey 08 540, USA

\begin{abstract}
We prove the positivity of the pressure and compressibility for neutral systems in the Thomas-Fermi theory of molecules. Our results include some new properties of the Thomas-Fermi potential and a proof that the kinetic energy is superadditive.
\end{abstract}

\section{Introduction}

The Thomas-Fermi (TF) theory of atoms, molecules and solids has been given a firm mathematical foundation and many of the qualitative properties of the theory are understood and have been proven [1] (see also [2]; properties of the manybody TF potential are proved in [3]). There were, however, some open questions in [1], one of which we solve in this paper: the positivity of the pressure and compressibly for neutral systems.

The TF theory is defined by the energy functional (in units in which $h^{2}(8 m)^{-1}(3 / \pi)^{2 / 3}=1$ and $|e|=1$, where $e$ and $m$ are the electron charge and mass)

$$
\begin{aligned}
\xi(\varrho) & =K(\varrho)-A(\varrho)+R(\varrho)+U \\
K(\varrho) & =\frac{3}{5} \int \varrho(x)^{5 / 3} d x \\
A(\varrho) & =\int V(x) \varrho(x) d x \\
V(x) & =\sum_{j=1}^{k} z_{j}\left|x-R_{j}\right|^{-1} \\
R(\varrho) & =\frac{1}{2} \iint \varrho(x) \varrho(y)|x-y|^{-1} d x d y \\
U & =\sum_{1 \leqq i<j \leqq k} z_{i} z_{j}\left|R_{i}-R_{j}\right|^{-1} .
\end{aligned}
$$

Here $z_{1}, \ldots, z_{k} \geqq 0$ are the charges of $k$ fixed nuclei located at $R_{1}, \ldots, R_{k} \cdot \int d x$ is always a three-dimensional integral. $\xi(\varrho)$ is defined for electron densities $\varrho(\mathrm{x}) \geqq 0$ such that $\int \varrho$ and $\int \varrho^{5 / 3}$ are finite.

* Work partially supported by U.S. National Science Foundation grant MCS 75-21684 A02

$\star \star$ On leave from Department of Physics, Universidad de Chile, Santiago, Chile 
The TF energy for $\lambda$ (not necessarily integral) electrons is defined by

$$
e(\lambda)=\inf \left\{\xi(\varrho) \mid \int \varrho=\lambda\right\}
$$

It is known [1] that for $\lambda \leqq Z \equiv \sum_{j=1}^{k} z_{j}$ there is a unique minimizing $\varrho$ for (1.3). It is the unique solution to the TF equation

$$
\varrho(x)^{2 / 3}=\max \left[\phi(\mathrm{x})-\Phi_{0}, 0\right]
$$

for some $\Phi_{0} \geqq 0$, and with

$$
\phi(x) \equiv V(x)-\int|x-y|^{-1} \varrho(y) d y .
$$

$-\Phi_{0}$ is the chemical potential [1], i.e.

$$
\frac{d e(\lambda)}{d \lambda}=-\Phi_{0}
$$

For $\lambda \leqq Z, \phi(x)>0$, all $x . \Phi_{0}=0$ if and only if $\lambda=Z$ and hence, for the neutral case the TF equation is

$$
\varrho^{2 / 3}(x)=\phi(x) \text {. }
$$

If $\lambda>Z$, there is no minimizing $\varrho$ for $(1.3)$, and $e(\lambda)=e(Z)$ in this case.

There are various possible definitions of the pressure. The one we shall use is the "change in energy under uniform dilation" defined as follows: Replace each $R_{i}$ by $l R_{i}, l$ being a scale factor, and let $e(\lambda, l)$ be the TF energy for a given $\lambda$ and $l$. Then $P=-\partial e / \partial V$ which we interpret as

$$
P=-\left(3 l^{2}\right)^{-1} \partial e(\lambda, l) / \partial l \text {. }
$$

The reciprocal compressibility, $\kappa^{-1}$, should be $-V \frac{\partial P}{\partial V}$ which we interpret as

$$
\kappa^{-1}=-(l / 3) \partial P / \partial l
$$

We shall prove that in the neutral case $P$ and $\kappa^{-1}$ are nonnegative (in the atomic case they are, of course, zero). In the process of doing so, we shall prove several interesting facts about the dependence of $\phi(x), K, A$ and $R$ on the $z_{i}$. (Note: here and in the sequel, $\phi(x), K, A, R$, etc. mean the respective quantities evaluated at the unique, minimizing TF density, $\varrho$.) We are not able to prove that $P$ and $\kappa$ are non-negative in the ionic (i.e. subneutral) case but conjecture that they are. The only thing we shall have to say about the ionic case except for appendix $B$ is to give a formula (1.14) for $P$ in terms of $e$ and $K$. We are led to make the further conjecture that $P$ is a decreasing function of $\lambda$ and thus that the neutral case is the worst case. When $\lambda=0, P>0$ and $\kappa>0$ because $e=l^{-1} \sum_{i<j} z_{i} z_{j}\left|R_{i}-R_{j}\right|^{-1}$. In other words, the pressure is positive because the nuclear repulsion dominates the attractive forces; this repulsion presumably grows stronger as electrons are removed from the system.

The above definitions $(1.6,1.7)$ of $P$ and $\kappa$ carry over, in the thermodynamic limit, to the ordinary definitions for a solid (see [1], Sect. VI). There are, however, 
two other useful definitions which we will not touch upon in the main text (see Appendix B, however) except to conjecture that $P$ and $\kappa$ are non-negative for these definitions as well.

(i) Dilation in one direction: Let $R_{i} \rightarrow\left(l R_{i}^{1}, R_{i}^{2}, R_{i}^{3}\right)$, instead of $R_{i} \rightarrow l R_{i}$. Since this is a one dimensional expansion it seems appropriate to define $P=-\partial e(\lambda, l) / \partial l$ and $\kappa^{-1}=-l \partial P / \partial l$. As far as nonnegativity is concerned, this new definition changes $\kappa$ but not $P$.

(ii) Separation relative to a plane: choose any plane which does not contain nuclei. For convenience it may be assumed to be the $x-y$ plane $\left\{\left(x^{1}, x^{2}\right.\right.$, $\left.\left.x^{3}\right) \mid x^{3}=0\right\}$. If $R_{i}=\left(R_{i}^{1}, R_{i}^{2}, R_{i}^{3}\right)$, replace $R_{i}^{3}$ by $R_{i}^{3}+l$ if $R_{i}^{3}>0$ and by $R_{i}^{3}-l$ if $R_{i}^{3}<0$. Note that in this case we shift by $l$ instead of dilate by $l$. Again, $P=-\partial e(\lambda, l) / \partial l$ and $\kappa^{-1}=-l \partial P / \partial l$. In appendix $B$ we will prove that $P>0$ if the plane is a symmetry plane. This latter case was also proved by Balàsz [4] but our proof is somewhat different; it uses reflection positivity. Balàsz assumed there were only two nuclei, but his method works for any symmetric situation. One reason for being interested in this special case is that our (and Balàsz') proofs are valid for the ionic case as well.

The definitions we shall work with $(1.6,1.7)$ have one virtue, namely the dependence of $e$ on $l$ can be converted into a dependence of $e$ on the $z_{i}$. This is a consequence of the following scaling properties:

Henceforth, $R_{1}, R_{2}, \ldots, R_{k}$ are fixed (with $R_{i} \neq R_{j}$ if $i \neq j$ ). We denote the $k$-tuple $z_{1}, \ldots, z_{k}$ simply by $\underline{z}$. Let $e(\underline{z}, \lambda, l)$ be the energy with the uniform dilation $l$. Then, from (1.1),

$$
e(\underline{z}, \lambda, l)=l^{-7} e\left(l^{3} \underline{z}, l^{3} \lambda, 1\right)
$$

and the minimizing TF density satisfies

$$
\varrho(\underline{z}, \lambda, l ; x)=l^{-6} \varrho\left(l^{3} \underline{z}, l^{3} \lambda, 1 ; x / l\right) .
$$

Substituting $(1.8)$ in $(1.6,1.7)$ yields (assuming that all derivatives exist)

$$
\begin{aligned}
& 3 l^{10} P=7 e-3 l^{3} \sum_{i=1}^{k} z_{i} e_{i}-3 l^{3} \lambda e_{2} \\
& 9 l^{10} \kappa^{-1}=70 e-42 l^{3} \sum_{i=1}^{k} z_{i} e_{i}-42 l^{3} \lambda e_{2} \\
& \quad+9 l^{6} \sum_{i, j=1}^{k} z_{i} z_{j} e_{i j}+9 l^{6} \lambda^{2} e_{22}+18 l^{6} \lambda \sum_{i=1}^{k} z_{i} e_{2 i} .
\end{aligned}
$$

In $(1.10,1.11)$ the notation is the following:

$$
e_{i} \equiv \partial e(\underline{x}, y, 1) / \partial x_{i}, \quad e_{2} \equiv \partial e(\underline{x}, y, 1) / \partial y, \quad \text { etc. }
$$

These quantities are evaluated at $\underline{x}=l^{3} \underline{z}$ and $y=l^{3} \lambda$. A numerical error in the expression for $\kappa^{-1}$ was made in Ref. [1], Eq. (145).

A more convenient form for $P$ is obtained by noting that $e=K-A+R+U$. Furthermore, if (1.4) is multiplied by $\varrho(x)$ and integrated over the set on which $\varrho(x) \geqq 0$, one obtains

$$
(5 / 3) K=A-2 R-l^{3} \lambda \Phi_{0}
$$


moreover, $e_{2}=-\Phi_{0}(\mathrm{cf} .(1.5))$. Finally,

$$
l^{3} \sum_{i=1}^{k} z_{i} e_{i}=2 U-A=2 e-(1 / 3) K+l^{3} \lambda \Phi_{0}
$$

([1], Theorem II.16 or Lemma V.7). Combining these facts and then using (1.8), yields, for all $\lambda$,

$$
3 l^{3} P(\underline{z}, \lambda, l)=e(\underline{z}, \lambda, l)+K(\underline{z}, \lambda, l) .
$$

For an atom, $2 K=A-R$ (Virial Theorem, [1], Theorem II.22), $U=0$ and $e=K-A+R$. Thus (1.14) gives $P=0$ for all $\lambda$, as it should in this case.

The conjecture stated above, that the neutral case is the worst can be given a more transparent form:

Conjecture 1. $e+K$ is a decreasing function of $\lambda$ for fixed $\underline{z}$ and $R_{i}$.

In this paper we will prove the positivity of $P$ and $\kappa$ for the neutral case. In the next Section the list of theorems to be proved is given. These theorems have an easy heuristic proof and these are given in Sect. III. We do so because these heuristic proofs are a guide to the proper proofs given in Sect. IV, and because they may be a useful guide to future work.

\section{Theorems to Be Proved}

We will be concerned only with the neutral case and use the notation $\phi(z, x), \varrho(\underline{z}, x)$, $e(\underline{z}), K(\underline{z}), A(\underline{z}), R(\underline{z}), U(\underline{z})$ to denote the TF potential and density at the point $x \in \mathbb{R}^{3}$, the total TF energy, the kinetic energy, the attractive energy, the electron repulsion and the nuclear repulsion, respectively, (cf. (1.1)), for the unique TF $\varrho$ that satisfies the TF equation $(1.4 \mathrm{~b}, 1.4 \mathrm{c}) . \underline{z} \in \mathbb{R}_{+}^{k} \equiv\left\{\left(z_{1}, \ldots, z_{k}\right) \mid z_{i} \geqq 0\right\}$. The $R_{i}$ are fixed and distinct.

Definitions. If $f$ is a real valued function on $\mathbb{R}_{+}^{k}$ then:

(i) $f$ is weakly superadditive (WSA) $\Leftrightarrow f\left(\underline{z}_{1}+\underline{z}_{2}\right) \geqq f\left(\underline{z}_{1}\right)+f\left(\underline{z}_{2}\right), \forall \underline{z}_{1}, \underline{z}_{2} \in \mathbb{R}_{+}^{k}$, such that $\left(\underline{z}_{1}, \underline{z}_{2}\right)=0$, i.e. $\left(z_{1}\right)_{i}\left(z_{2}\right)_{i}=0, \forall i$.

(ii) $f$ is superadditive (SA) $\Leftrightarrow f\left(\underline{z}_{1}+\underline{z}_{2}\right) \geqq f\left(\underline{z}_{1}\right)+f\left(\underline{z}_{2}\right), \forall \underline{z}_{1}, \underline{z}_{2} \in \mathbb{R}_{+}^{k}$.

(iii) $f$ is strongly superadditive (SSA) $\Leftrightarrow f\left(\underline{z}_{1}+\underline{z}_{2}+\underline{z}_{3}\right)-f\left(\underline{z}_{1}+\underline{z}_{2}\right)-f\left(\underline{z}_{1}+\underline{z}_{3}\right)$ $+f\left(\underline{z}_{1}\right) \geqq 0, \forall \underline{z}_{1}, \underline{z}_{2}, \underline{z}_{3} \in \mathbb{R}_{+}^{k}$.

(iv) $f$ is ray convex $\Leftrightarrow f\left(\lambda \underline{z}_{1}+(1-\lambda) \underline{z}_{2}\right) \leqq \lambda f\left(\underline{z}_{1}\right)+(1-\lambda) f\left(\underline{z}_{2}\right), \forall \lambda \in[0,1], \underline{z}_{1}$, $\underline{z}_{2} \in \mathbb{R}_{+}^{k}$ and either $\underline{z}_{1}-\underline{z}_{2} \in \mathbb{R}_{+}^{k}$ or $\underline{z}_{2}-\underline{z}_{1} \in \mathbb{R}_{+}^{k}$.

(v) $f$ is ray concave $\Leftrightarrow-f$ is ray convex.

(vi) $f$ is increasing $\Leftrightarrow f\left(\underline{z}_{1}+\underline{z}_{2}\right) \geqq f\left(\underline{z}_{1}\right), \forall \underline{z}_{1}, \underline{z}_{2} \in \mathbb{R}_{+}^{k}$.

Obviously,

$f$ is SSA and $f(0) \leqq 0 \Rightarrow f$ is $\mathrm{SA} \Rightarrow f$ is WSA.

Further relations among these definitions are proved in Appendix A. These are the following $\left(C^{p}\left(\mathbb{R}_{+}^{k}\right)\right.$ denotes the $p$-fold continuously differentiable functions and subscripts denote partial derivatives):

Lemma 2.1. (i) If $f \in C^{2}\left(\mathbb{R}_{+}^{k}\right)$ then $f$ is $S S A \Leftrightarrow f_{i j} \geqq 0, \forall i, j$.

(ii) If $f \in C^{1}\left(\mathbb{R}_{+}^{k}\right)$ then $f$ is $\mathrm{SSA} \Leftrightarrow f_{i}$ is increasing. 
Lemma 2.2. (i) If $f \in C^{2}\left(\mathbb{R}_{+}^{k}\right), f(0)=0$, and $f_{i j} \geqq 0 \forall i \neq j$, then $f$ is WSA. WSA.

(ii) If $f \in C^{1}\left(\mathbb{R}_{+}^{k}\right), f(0)=0$ and $f_{i}$ is an increasing function of $z_{j}$ for $j \neq i$, then $f$ is

Remark. The converse implication is false as the WSA function $f(z)=z_{1} \sin ^{2}\left(z_{2}\right)$ on $\mathbb{R}_{+}^{2}$ shows.

Lemma 2.3. $f$ is SSA implies $f$ is weakly ray-convex, i.e. $f$ satisfies definition (iv) with $\lambda=1 / 2$.

Remark. The converse implication is false, even if SSA is replaced by WSA. On $\mathbb{R}_{+}^{2}$, $f(z)=\left|z_{1}-z_{2}\right|+z_{1}+z_{2}$ is convex (not merely ray-convex), increasing and $f(0)=0$, but $f(1,1)<f(1,0)+f(0,1)$.

Lemma 2.4. If $f$ is ray-convex and $f \in C^{1}\left(\mathbb{R}_{+}^{k}\right)$ then $f_{i}$ is increasing.

Corollary 2.5. If $f$ is ray-convex and $f \in C^{1}\left(\mathbb{R}_{+}^{k}\right)$ then

$$
\sum_{i=1}^{k} z_{i}^{\prime} f_{i}(\underline{z}) \leqq f\left(\underline{z}+\underline{z}^{\prime}\right)-f(\underline{z}) \leqq \sum_{i=1}^{k} z_{i}^{\prime} f_{i}\left(\underline{z}+\underline{z}^{\prime}\right) .
$$

The theorems to be proved can now be stated.

Properties of $\phi(z, x)$ (neutral case):

Theorem 2.6. For each fixed $x \in \mathbb{R}^{3}$, different from $R_{1}, \ldots, R_{k}, \underline{z} \mapsto \phi(\underline{z}, x)$ is in $C^{1}\left(\mathbb{R}_{+}^{k}\right)$ and $C^{2}\left(\mathbb{R}_{+}^{k}\lfloor\underline{0})\right.$. $\underline{z} \mapsto \phi_{i}(\underline{z}, x)$ and $\underline{z} \mapsto \phi_{i j}(\underline{z}, x)$ are equicontinuous in $x$. Furthermore,

(i) $\phi_{i j}(z, x) \leqq 0, \forall i, j$, and is negative semidefinite as a matrix, i.e. $\sum_{i, j=1}^{k} \bar{c}_{i} c_{j} \phi_{i j}(z, x)$ $\leqq 0$ for all $\underline{c} \in C^{k}$.

(ii) $\phi_{i j}\left(\underline{z}, R_{p}\right)=\lim _{x \rightarrow R_{p}} \phi_{i j}(\underline{z}, x)$ exists $(\underline{z} \neq \underline{0})$.

(iii) $\underline{z} \mapsto \phi_{i}(\underline{z}, x) \geqq 0$ and is ray-convex, $\forall i$.

(iv) $\lim _{x \rightarrow R_{2}}\left\{\phi_{i}(z, x)-\left|x-R_{i}\right|^{-1}\right\} \leqq 0$ exists. $\phi_{i}(z, x)<\left|x-R_{i}\right|^{-1}$.

(v) $\phi_{i}\left(z, R_{j}\right)=\lim _{x \rightarrow R_{j}} \phi_{i}(z, x)$ exists for $i \neq j$. Moreover, $\phi_{i}\left(z, R_{j}\right)=\phi_{j}\left(z, R_{i}\right)$.

(vi) For every $\alpha<(1+\sqrt{73}) / 2$, there exist an $R(\alpha)<\infty$ and finite numbers $M(\alpha)$ and $B(\alpha)$ such that $\phi_{i}(\underline{z}, x) \leqq M(\alpha)|x|^{-\alpha},-\phi_{i j}(\underline{z}, x) \leqq B(\alpha)|x|^{-\alpha},(\underline{z} \neq 0)$, hold when $|x|$ $>R(\alpha)$.

Using Lemma 2.1, we have

Corollary 2.7. For each fixed $x \in \mathbb{R}^{3}$, different from $R_{1}, R_{2}, \ldots, R_{k}$,

(i) $-\phi(z, x)$ is SSA.

(ii) $\phi(\underline{z}, x)$ is concave (not merely ray-concave).

(iii) $-\phi_{i}(z, x)$ and $(\phi(\underline{z}, x)$ are strictly increasing.

Remark. That $\phi(z, x)$ is increasing is Teller's Lemma [5], [Theorem V.5, [1]]. 
Properties of $K, A, R$ and e (neutral case):

Theorem 2.8. $K(\underline{z}) \in C^{1}\left(\mathbb{R}_{+}^{k}\right)$ and $C^{2}\left(\mathbb{R}_{+}^{k}\lfloor\underline{0})\right.$ and :

(i) $K_{i}(\underline{z})=3 \lim _{x \rightarrow R_{i}}\left[\phi(\underline{z}, x)-\sum_{j=1}^{k} z_{j} \phi_{j}(\underline{z}, x)\right]$

(ii) $K_{i j}(\underline{z})=-3 \sum_{p=1}^{k} z_{p} \phi_{i j}\left(\underline{z}, R_{p}\right)$.

Remark. The limit in (2.2) exists by Theorem 2.6, and by $e_{i}=\lim _{x \rightarrow R_{i}}\{\phi(z, x)$ $\left.-z_{i}\left|x-R_{i}\right|^{-1}\right\}([1]$, Theorem II.16, Lemma V.7).

Using Theorem 2.6 we have

Corollary 2.9. (i) $K_{i j}(z) \geqq 0$ and is positive semidefinite as a matrix,

(ii) $K(z)$ is convex (not merely ray-convex) and SSA on $\mathbb{R}_{+}^{k}$,

(iii) $K(\underline{0})=0$, which implies $K(\underline{z})$ is SA.

Theorem 2.10. (i) $R(\underline{z})$ and $A(z)$ are convex (not merely ray convex) and SSA on $\mathbb{R}_{+}^{k}$. (ii) $e(\underline{z})$ is WSA on $\mathbb{R}_{+}^{k}$.

Remark. (ii) is just Teller's Theorem [5], [1, Theorem V.1], $e(\underline{z})$ is not SA. For $k=1$, $e(\underline{z})=-$ (const.) $z^{7 / 3},[1]$, and this is not SA. However we make the following.

Conjecture 2. Let $\tilde{e}(z)=\sum_{j=1}^{k} e^{a t}\left(z_{j}\right)$, where $e^{a t}(z)$ is the TF energy of an isolated atom of charge $z$. Then $e(\underline{z})-\tilde{e}(z)$ is SA.

Remark. $e-\tilde{e}$ is not SSA because

$$
\left(\partial^{2} / \partial z_{i}^{2}\right)(e-\tilde{e})=\lim _{x \rightarrow R_{i}}\left(\phi_{i}(\underline{z}, x)-\left(\partial \phi^{a t} / \partial z\right)\left(z_{i}, x\right)\right),
$$

and this is negative if some $z_{j} \neq 0(j \neq i)$ by Corollary 2.7 (iii). It is obvious that $e-\tilde{e}$ is WSA since $e$ and $-\tilde{e}$ are both WSA.

Definition. $X(\underline{z}) \equiv 3 K(\underline{z})-\sum_{i=1}^{k} z_{i} K_{i}(\underline{z})$

Theorem 2.11. $X(\underline{z})$ is SSA and $X(\underline{0})=0$. Hence $X$ is SA. Moreover $X(\underline{z})$ is ray convex (as follows from Lemma 2.3 and Theorem 2.8).

These theorems can be combined to yield the desired results about the pressure and compressibility.

Theorem 2.12. For the neutral molecule, the pressure and compressibility as given by (1.6), (1.7) exist and satisfy:

(i) $3 l^{3} P(\underline{z})=e(\underline{z})+K(\underline{z})$,

(ii) $9 l^{3} \kappa^{-1}(\underline{z})=6 l^{3} P(\underline{z})+2 e(\underline{z})+3 X(\underline{z})(c f .(2.4))$,

(iii) $P$ and $\kappa^{-1}$ are WSA and non-negative,

(iv) $l^{2} P(\underline{z}, l)$ is a decreasing function of $l$. Equivalently, $e(\underline{z}, l)$ is a convex function of $l$. 
Proof. We can write (1.8) in the form $e(\underline{z}, l)=l^{-7} e\left(l^{3} \underline{z}\right)$, where $e\left(l^{3} \underline{z}\right) \equiv e\left(l^{3} \underline{z}, 1\right)$. Hence $3 l^{10} P=7 e-3 l^{3} \sum_{i=1}^{k} z_{i} e_{i}$ since $z_{i} e_{i}$ exists [1]. (1.12), (1.13) are true [1], and so (2.5) is proved [cf.(1.14)]. Since $e$ and $K$ are WSA, so is $e+K$ and $e+K \geqq$ (sum of the $e+K$ for isolated atoms $)=0$. Using scaling again on the right side of (2.5) $\left(K(\underline{z}, l)=l^{-7} K\left(l^{3} \underline{z}, 1\right)\right.$ also), and Theorem 2.8(i), we can differentiate (2.5). Again using (1.13) and rescaling, (2.6) is obtained. By Theorem 2.11, (iii) is true. To prove (iv) note that for an atom, $e=-$ (const.) $z^{7 / 3}$ and $K=-e$; hence $2 e+3 X=0$ in this case. Since $e$ and $X$ are WSA, $2 e+3 X \geqq 0$. Thus $-l \frac{\partial P}{\partial l} \geqq 2 P$. If one writes $P(\underline{z}, l)$ $=l^{-7} \pi(\underline{z}, l)$, then $\partial \pi / \partial l \leqq 0$.

The following conjecture, if true, would show that $l^{4} P$ is decreasing, for the right side of $(2.6)$ is $12 l^{3} P(\underline{z})+\tilde{X}(z)$. It would also show that $K(z, l)$ is decreasing in $l$.

Conjecture 3. $\tilde{X}(\underline{z}) \equiv 3 X(\underline{z})-2 K(\underline{z})$ is WSA.

Remark. $\tilde{X}(z)=0$ for an atom.

Let us define $E(z)=e(\underline{z})-U(\underline{z})$. It has been proved $([1]$, Theorem V.3) that $-E(z)$ is WSA. We conjecture that something stronger holds, namely

Conjecture 4. $E(\underline{z}, l)$ is monotone increasing in $l$, for fixed $\underline{z}$.

Remark. It is easy to check that Conjecture 4 is implied by Conjecture 1 . Conjecture 4 means that the pressure of a molecule in which the nuclear-nuclear repulsion is neglected is negative instead of positive. Some results in this direction for the Schrödinger theory are given in [11].

\section{Heuristic Proofs}

In this section we give simple, but non-rigorous proofs that $K(\underline{z})$ and $X(\underline{z}),(2.4)$, are SSA and $K(\underline{z})$ is convex. From this, Theorem 2.12 on the positivity of $P$ and $K$ follows, as mentioned in Sect. II. We think it is important to provide these "proofs" because the main line of the argument may be obscure in the proper proofs given in the next section. These "proofs" assume that all necessary derivatives exist. Let us begin with some facts about the TF potential $\phi(z, x)$. Hereafter we refer only to the neutral case. By $(1.4) \phi(z, x)$ satisfies the TF equation

$$
-(4 \pi)^{-1} \Delta \phi(\underline{z}, x)+\phi(\underline{z}, x)^{3 / 2}=\sum_{i=1}^{k} z_{i} \delta\left(x-R_{i}\right) .
$$

The kernel for $\left[-(4 \pi)^{-1} \Delta+\phi^{1 / 2}\right]^{-1}$ is positive, and $z_{i} \delta\left(x-R_{i}\right)$ are positive "functions". Therefore $\phi(\underline{z}, x) \geqq 0$ all $x$. Differentiating (3.1) twice with respect to the $z$ 's we formally get

$$
\left[-(4 \pi)^{-1} \Delta+(3 / 2) \phi(\underline{z}, x)^{1 / 2}\right] \phi_{i}(\underline{z}, x)=\delta\left(x-R_{i}\right)
$$

and

$$
\left[-(4 \pi)^{-1} \Delta+(3 / 2) \phi(\underline{z}, x)^{1 / 2}\right] \phi_{i j}(\underline{z}, x)=-(3 / 4) \phi(\underline{z}, x)^{-1 / 2} \phi_{i}(\underline{z}, x) \phi_{j}(\underline{z}, x) .
$$


From Eq. (3.2a) we have $\phi_{i} \geqq 0$, since the kernel for $\left[-(4 \pi)^{-1} \Delta+(3 / 2) \phi^{1 / 2}\right]^{-1}$ is positive. For the same reason, $\phi_{i j} \leqq 0$, all $i, j$, and therefore $-\phi$ is SSA. Multiplying (3.2b) by $\bar{c}_{i}, c_{j}$, with $c_{j} \in C$, and summing over $i, j$ we get,

$$
\left[-(4 \pi)^{-1} \Delta+(3 / 2) \phi(\underline{z}, x)^{1 / 2}\right] \sum_{i, j=1}^{k} \bar{c}_{i} \phi_{i j} c_{j}=-(3 / 4) \phi^{-1 / 2}\left|\sum_{i=1}^{k} c_{i} \phi_{i}\right|^{2} .
$$

Therefore the quadratic form $\sum_{i, j=1}^{k} \bar{c}_{i} \phi_{i j}(\underline{z}, x) c_{j}$ is non-positive for all $\underline{c} \in C^{k}$. Hence $\phi$ is concave in $\mathbb{R}_{+}^{k}$. Finally differentiating (3.2b) with respect to $z_{l}$ we have,

$$
\begin{aligned}
& {\left[-(4 \pi)^{-1} \Delta+(3 / 2) \phi^{1 / 2}\right] \phi_{i j l}} \\
& \quad=(3 / 8) \phi^{-3 / 2} \phi_{i} \phi_{j} \phi_{l}-(3 / 4) \phi^{-1 / 2}\left[\phi_{i l} \phi_{j}+\phi_{i j} \phi_{l}+\phi_{j l} \phi_{i}\right]
\end{aligned}
$$

which in turn implies $\phi_{i j l} \geqq 0$ all $i, j, l$. Indeed the following is formally true: $(-1)^{n+1} \phi_{i_{1} i_{2} \ldots i_{n}} \geqq 0$ for all $i_{j}$ and all $n \geqq 1$.

Remark. If one assumes that the derivative $\phi_{i_{1} \ldots i_{n}}$ exists, then Theorem 4 of Ref. [3] shows that the sign is indeed $(-1)^{n+1}$. To use Theorem 4 for this purpose it is necessary to choose $R_{i}=R_{j}$ for some $i \neq j$, but this is allowed, as explained in [3] in the paragraph after (1.6). Theorem 4 of [3] directly gives the SSA of $-\phi$ without going through Lemma 2.1. Indeed, Theorem 4 is a generalization of SSA; for example $\phi\left(\underline{z}_{1}+\underline{z}_{2}+\underline{z}_{3}+\underline{z}_{4}, x\right)-\phi\left(\underline{z}_{1}+\underline{z}_{2}+\underline{z}_{3}, x\right)-\phi\left(\underline{z}_{1}+\underline{z}_{3}+z_{4}, x\right)-\phi\left(\underline{z}_{1}+\underline{z}_{2}\right.$ $\left.+\underline{z}_{4}, x\right)+\phi\left(\underline{z}_{1}+\underline{z}_{2}, x\right)+\phi\left(\underline{z}_{1}+\underline{z}_{3}, x\right)+\phi\left(\underline{z}_{1}+\underline{z}_{4}, x\right)-\phi\left(\underline{z}_{1}, x\right) \geqq 0$. However, we are obliged to prove the existence of the first two derivatives of $\phi(z, x)$ because we need them in our proof that $K(z)$ and $X(z)$ are SSA.

From $\phi_{i j l} \geqq 0$ follows the ray-convexity of $\phi_{i}$ because the quadratic form $\sum_{j, l=1}^{k}\left(\phi_{i}\right)_{j l} z_{j} z_{l}$ is non-negative for all $\underline{z} \in \mathbb{R}_{+}^{k}$.

Now, let us formally show that $K$ is SSA. We have to prove that $K_{i j} \geqq 0$ all $i, j$ (see Lemma 2.1). For the neutral molecule the kinetic energy is given by

$$
K(\underline{z})=(3 / 5) \int \phi(\underline{z}, x)^{5 / 2} d x .
$$

Differentiating (3.3) twice with respect to the $z$ 's we get,

$$
\begin{aligned}
K_{i j}(\underline{z})= & (3 / 2)\left[\int \phi(\underline{z}, x)^{3 / 2} \phi_{i j}(\underline{z}, x) d x\right. \\
& \left.+(3 / 2) \int \phi(\underline{z}, x)^{1 / 2} \phi_{i}(\underline{z}, x) \phi_{j}(\underline{z}, x) d x\right] .
\end{aligned}
$$

Introducing (3.2b) in (3.4), partial integration yields

$$
K_{i j}=3 \int \phi_{i j}\left((4 \pi)^{-1} \Delta \phi-\phi^{3 / 2}\right) d x=-3 \sum_{l=1}^{k} z_{l} \phi_{i j}\left(R_{l}\right),
$$

where the last equality is a consequence of (3.1). But $\phi_{i j} \leqq 0$, all $i, j$ and therefore $K_{i j} \geqq 0$ and $K$ is SSA. Furthermore $\left[\phi_{i j}\right]$ is negative semi-definite [recall the discussion after $(3.2 \mathrm{~b})]$; hence $\left[K_{i j}\right]$ is positive semi-definite and $K$ is convex. 
It remains to be shown that $X_{i j} \geqq 0$, all $i, j$, that is, $X$ is SSA (Lemma 2.1). Differentiating $X$ twice with respect to $z_{i}, z_{j}$ we have

$$
\begin{aligned}
X_{i j} & =K_{i j}-\sum_{l=1}^{k} z_{l} K_{i j l} \\
& =3 \sum_{l, m=1}^{k} z_{l} z_{m} \phi_{i j m}\left(R_{l}\right),
\end{aligned}
$$

where the last equality follows from (3.5). Therefore $X_{i j} \geqq 0$, all $i, j$ because $\phi_{i j m}(x) \geqq 0 \forall x, i, j, m$.

\section{Proof of Theorems 2.6-2.11}

Here we give the rigorous proofs of the theorems enunciated in Sect. II. Only neutral systems are considered. Let us begin by recalling some of the known facts about the TF potential $\phi(z, x)$ that we are going to need in our proofs:

$(\mathrm{P}-1) \phi(\underline{z}, x)$ satisfies,

$$
\phi(\underline{z}, x)=\sum_{i=1}^{k} z_{i}\left|x-R_{i}\right|^{-1}-\int|x-y|^{-1} \phi(\underline{z}, y)^{3 / 2} d y .
$$

(P-2) $\phi(\underline{z}, x)$ is bounded and continuous on any open subset of $\mathbb{R}^{3}$ which is at non-zero distance from all the $R_{i}([1]$, Theorem IV.1). In fact, the TF potential is real analytic away from all the $R_{j}$, on all of $\mathbb{R}^{3}$ ([1], Theorem IV.6). $\left(\phi(\underline{z}, x)-\sum_{i=1}^{k} z_{i}\left|x-R_{i}\right|^{-1}\right.$ is continuous for all $x$. $)$

(P-3) $\phi(\underline{z}, x)$ is strictly positive for $\underline{z} \neq \underline{0}$ ([1], Theorem IV.3).

(P-4) $|x|^{4} \phi(z, x) \rightarrow 9 \pi^{-2}$ as $|x| \rightarrow \infty$, uniformly with respect to direction. (This is Sommerfeld's . formula, [1], Theorem IV.10.) Moreover, for every $c<3 \pi^{-1} \exists R(c)<\infty$ such that $\phi(z, x) \geqq c^{2}|x|^{-4}$ when $|x| \geqq R(c)([1]$, Theorems IV.8, IV.10).

(P-5) Properties (P-1) and (P-2) imply that $\phi(\underline{z}, x)=z_{j}\left|x-R_{j}\right|^{-1}+g(x)$ near $R_{j}$, where $g$ is a continuous function.

(P-6) By the foregoing $\phi(z, x) \in L^{p}$ for every $p \in[1,3)$, and $\phi(z, x)^{1 / 4} \in L^{p}$ for every $p \in(3,12)$.

(P-7) $\phi(\underline{z}, x)$ is increasing in $\underline{z}$ for every $x \in \mathbb{R}^{3}$. (This is Teller's lemma, [1], Theorem V.5.)

(P-8) $\phi(\underline{z}, x)$ is strongly subadditive in $\underline{z}$ for every $x \in \mathbb{R}^{3}$ ([3], Theorem 4$)$. In particular $\phi(z, x)$ is subadditive.

(P-9) $\phi(z, x)$ is concave in $\underline{z}$.

Proof of (P-9). Let $\psi(x) \equiv\left(\phi(\underline{z}, x)-\alpha \phi\left(\underline{z}_{1}, x\right)-(1-\alpha) \phi\left(\underline{z}_{2}, x\right)\right.$, with $\underline{z} \equiv \alpha \underline{z}_{1}$ $+(1-\alpha) \underline{z}_{2}, 0 \leqq \alpha \leqq 1$. By (P-2) $\psi$ is continuous for all $x$ and by (P-4) $\psi$ goes to zero at infinity, hence $S=\{x \mid \psi(x)<0\}$ is open and $\psi=0$ on $\partial S \cup\{\infty\}$. On $S,-(4 \pi)^{-1} \Delta \psi$ $=-\phi(\underline{z}, x)^{3 / 2}+\alpha \phi\left(\underline{z}_{1}, x\right)^{3 / 2}+(1-\alpha) \phi\left(\underline{z}_{2}, x\right)^{3 / 2} \geqq-\phi(\underline{z}, x)^{3 / 2}+\phi(\underline{z}, x)^{3 / 2}=0$ because $t \mapsto t^{3 / 2}$ is convex. "Hence $\psi$ is superharmonic on $S$ and thus $\psi$ takes its minimum on $\partial S \cup\{\infty\}$ where it is zero. Then $S$ is empty." 
Remark. Since the argument between the apostrophes in the last paragraph repeats several times throughout this paper we will denote it by MMP (maximum modulus principle) to abbreviate.

If we call $\phi_{i}(\underline{z}, x)$ the derivative of $\phi(\underline{z}, x)$ with respect to $z_{i}$, we have formally

$\phi_{i}(z, x)=\left|x-R_{i}\right|^{-1}-\frac{3}{2} \int|x-y|^{-1} \phi(z, y)^{1 / 2} \phi_{i}(z, y) d y$.

Our first task will be to investigate the general properties of equations like (4.2).

\section{IV.1. General Properties of an Integral Equation [Eq. (4.4)]}

We deal here with $L^{p}$ spaces ( $\mathbb{R}^{3}$ always being understood) and with the weak $L_{w}^{p}$ spaces:

Definition. $f \in L_{w}^{p}(p>0)$ if and only if there is a constant $c<\infty$ such that $D_{f}(a) \equiv \mu\{x|| f(x) \mid>a\} \leqq c^{p} a^{-p}$, all $a>0$, where $\mu$ is Lebesgue measure. The infimum of all such $c$ is denoted by $\|f\|_{p, w}$.

Remarks. (1) $L^{p} C L_{w}^{p}$ (for $p \geqq 0$ ). If $f \in L^{p},\|f\|_{p, w} \leqq\|f\|_{p}$.

(2) $\|f\|_{p, w}$ is not a norm since it fails to satisfy the triangle inequality.

(3) It can be easily checked from the definition that $|x|^{-1} \in L_{w}^{3}$.

We will need the following later.

Lemma 4.1. If $f, g \in L_{w}^{p}$ and $L_{w}^{q}$ respectively then $f \cdot g \in L_{w}^{r}$ and $\|f \cdot g\|_{r, w}$ $\leqq 2^{1 / r}\|f\|_{p, w}\|g\|_{q, w}$, with $r^{-1}=p^{-1}+q^{-1}$ and $0<p, q, r<\infty$.

Proof. Without loss we can assume $\|f\|_{p, w}=\|g\|_{q, w}=1$.

$$
\{x|| f(x) g(x) \mid>a\} \subset\left\{x|| f(x) \mid>a^{r / p}\right\} \cup\left\{x|| g(x) \mid>a^{r / q}\right\} .
$$

Therefore $D_{f g}(a) \leqq D_{f}\left(a^{r / p}\right)+D_{g}\left(a^{r / q}\right)$. But $D_{f}\left(a^{r / p}\right) \leqq a^{-r}\|f\|_{p, w}^{p}$ and $D_{g}\left(a^{r / q}\right)$ $\leqq a^{-r}\|g\|_{q, w}^{q}$, whence $D_{f g}(a) \leqq 2 a^{-r}$.

Notes. (i) The constant $2^{1 / r}$ is not the best possible. It is easy to find a better one, namely $p^{1 / p} q^{1 / q} / r^{1 / r}$.

(ii) For more details about $L_{w}^{p}$ spaces the reader can consult [6].

The main tool to show existence and uniqueness of solutions to equations like (4.2) in some function spaces is given by:

Theorem 4.2. Let $w \in L_{w}^{3}\left(\mathbb{R}^{3}\right), w$ real, and let $\frac{3}{2}<p<3$. Then the map

$$
T_{w}: g \mapsto w(x) \int|x-y|^{-1} w(y) g(y) d y,
$$

is a bounded map from $L^{p}\left(\mathbb{R}^{3}\right) \rightarrow L^{p}\left(\mathbb{R}^{3}\right)$.

Note. Theorems of this kind have been proved by Faris [9] and Strichartz [10].

Proof. By the previous lemma $A_{w}: g \mapsto w g$ is a bounded map from $L_{w}^{p} \rightarrow L_{w}^{r}$ with $r^{-1}=p^{-1}+1 / 3$. Also $A_{w}$ restricted to $L^{p}$ is a bounded map by Remark (1). Now, $B: h \mapsto|x|^{-1} * h$ is a bounded map from $L_{w}^{r} \rightarrow L_{w}^{t}$ with $1+t^{-1}=r^{-1}+(1 / 3)$ (since $|x|^{-1} \in L_{w}^{3}$, and the weak form of Young's inequality, [6]), when $t>1,1<r<3 / 2$. Therefore $T_{w}=A_{w} B A_{w}$ is a bounded map from $L_{w}^{p} \rightarrow L_{w}^{p}$ for all $p \in(3 / 2,3)$. Finally by the Marcinkiewicz-Zygmund interpolation theorem $T_{w}$ extends to a bounded map from $L^{p} \rightarrow L^{p}, 3 / 2<p<3$. 
If we restrict the domain of $T_{w}$ to $L^{2}, T_{w}$ is a bounded operator from the Hilbert space $L^{2}$ into itself. Moreover $T_{w}$ is self-adjoint and positive since the kernel $|x-y|^{-1}$ is positive definite. Hence we have,

Corollary 4.3. The equation $\left(T_{w}+1\right) g=u$ with $T_{w}$ defined by (4.3) and $w \in L_{w}^{3}, u \in L^{2}$ has a unique $L^{2}$ solution, $g$.

We now obtain the main result of this section:

Theorem 4.4. Let $w \in L_{w}^{3}$ and $w v \in L^{2}$. Then there is a unique $f$ (defined a.e.) which satisfies the equation

$$
f(x)=v(x)-\int|x-y|^{-1} w(y)^{2} f(y) d y
$$

a.e. and such that $w f \in L^{2}$.

Proof. (i) Existence: Define

$$
f(x) \equiv v(x)-\int|x-y|^{-1} w(y) g(y) d y,
$$

where $g$ is the unique $L^{2}$ solution (by Corollary 4.3) to

$$
\left(T_{w}+1\right) g=v w .
$$

From (4.5) and (4.6) we have $w f=g$ and therefore the $f$ defined in this way satisfies (4.4) and also $w f \in L^{2}$. (ii) Uniqueness: Assume there are two solutions $f_{1}, f_{2}$ to Eq. (4.4) such that $w f_{1}$ and $w f_{2} \in L^{2}$. Both $w f_{1}$ and $w f_{2}$ satisfy Eq. (4.6) which has a unique solution. Therefore $w f_{1}=w f_{2}$ a.e., and hence $f_{1}=f_{2}$ a.e. [using (4.4)].

Having shown the existence of a unique solution to (4.4) for some class of $v$ and $w$, we next specialize to the particular $v$ of interest.

Theorem 4.5. Let $u \in \mathbb{R}^{3}$ and let $f_{u}$ be the solution to the equation

$$
f_{u}(x)=|x-u|^{-1}-\int|x-y|^{-1} w(y)^{2} f_{u}(y) d y,
$$

with $w \in L_{w}^{3}$ and $h_{u}(x) \equiv w(x)|x-u|^{-1} \in L^{2}\left(\mathbb{R}^{3}\right)$ for all $u \in \mathbb{R}^{3}$. Then the integral in (4.7) is finite for all $x \in \mathbb{R}^{3}$ and thus $f_{u}(x)$ is defined by the right side of (4.7) for all $x \neq u$. Furthermore, if $u \neq t \in \mathbb{R}^{3}, f_{u}(t)=f_{t}(u)$.

Proof. Since $w \in L_{w}^{3}$ and $h_{u}(x) \in L^{2}$. Theorem 4.4 implies that there is a unique (a.e.) function solving (4.7) and satisfying $w f_{u} \equiv g_{u} \in L^{2}$. Since $w f_{u} \in L^{2}$ and $h_{x}(y) \in L^{2}$ for all $x$, the integrand in (4.7) is absolutely integrable for all $x$. Now, $g_{u}$ satisfies $\left(1+T_{w}\right) g_{u}=h_{u}$ and $\left(g_{t}, h_{u}\right)=\left(g_{t},\left(1+T_{w}\right) g_{u}\right)=\left(\left(1+T_{w}\right) g_{t}, g_{u}\right)=\left(h_{t}, g_{u}\right)$ since $T_{w}$ [defined in (4.3)] is self-adjoint. Explicitly, this says

$$
\int w(x)^{2}\left[f_{t}(x)|x-u|^{-1}-f_{u}(x)|x-t|^{-1}\right] d x=0 .
$$

Using (4.7) this implies $0=f_{u}(t)-|t-u|^{-1}-f_{t}(u)+|u-t|^{-1}$.

Up to now the only assumption on $w$ was $w \in L_{w}^{3}$. We will now make a stronger assumption about $w$ in order to obtain continuity of the solution to (4.7). First, a preliminary remark : 
Lemma 4.6. If $f \in L^{p}$ and $g \in L^{q}$ with $p, q$ dual indices different from 1 and $\infty$ then $f * g$ is a bounded continuous function going to zero at infinity.

Proof. This result is standard. See [1], Lemma II.25.

Lemma 4.7. Let $w \in L_{w}^{3}$ and such that $w \in L^{6-\varepsilon} \cap L^{6+\varepsilon}$ (for some $\varepsilon>0$ ). Let $v$ be such that $v w \in L^{2}$ and let $f$ denote the solution to (4.4). Then the integral in (4.4) (namely $f-v)$ is a bounded continuous function going to zero at infinity.

Proof. By Theorem 4.4 the solution $f$ exists and satisfies $g \equiv w f \in L^{2}$. Using Hölder's inequality, $w g \in L^{p_{-}} \cap L^{p_{+}}$with $p_{+}=2(6 \pm \varepsilon)(8 \pm \varepsilon)^{-1}$. We can always decompose $|x|^{-1}=|x|_{<}^{-1}+|x|_{>}^{-1}$ with $|x|_{<}^{-1} \in L^{3-\eta_{+}},|x|_{>}^{-1} \in L^{3+\eta_{-}} \quad\left(\eta_{ \pm}\right.$, positive). Choose $\eta_{ \pm}=\varepsilon(4 \pm \varepsilon)^{-1}$; then $\left(3 \mp \eta_{ \pm}\right)$is the dual of $p_{ \pm}$. But $f-v=|x|^{-1} * g w$ $=|x|_{<}^{-1} * g w+|x|_{>}^{-1} * g w$, and hence this lemma follows from Lemma 4.6.

Remark. The $w$ which will eventually be used is simply $\phi^{1 / 4}(\underline{z}, x)$. This satisfies the conditions of Lemma 4.7 by (P-6).

We now study the dependence of the solution $f$ on $v$ and $w$.

Lemma 4.8. Let $w \in L_{w}^{3}$ and such that $w \in L^{6-\varepsilon} \cap L^{6+\varepsilon}$ (for some $\varepsilon>0$ ). Let $u \in \mathbb{R}^{3}$ be a parameter, and let

$$
v_{u}(x)=|x-u|^{-1}+V(x)
$$

where $V(x)$ is a continuous superharmonic function, bounded and going to zero at infinity such that $w V \in L^{2}$, then:

(i) The solution $f_{u}$ to (4.4) is non-negative for all $x$.

(ii) If $v_{u}$ is fixed and if $w_{1}(x)^{2} \geqq w_{2}(x)^{2}$ all $x$, the corresponding solutions $f_{1}$, (resp. $f_{2}$ ) to (4.4) with $w=w_{1}$ (resp. $w=w_{2}$ ) are such that $f_{1}(x) \leqq f_{2}(x)$ all $x$.

(iii) Now keep $w$ fixed. Let $v_{1 u}, v_{2 u}$ be of the form (4.8) with $v_{1 u}-v_{2 u}$ superharmonic, then the corresponding solutions $f_{1 u}, f_{2 u}$ are such that $f_{1 u}(x) \geqq f_{2 u}(x)$ all $x$.

Proof. Since $w \in L^{6-\varepsilon} \cap L^{6+\varepsilon}$ and $|x-u|^{-1} \in L^{3-\eta+}+L^{3+\eta-}$ with $\eta_{ \pm}=\varepsilon(4 \pm \varepsilon)^{-1}$, using Hölder's inequality we have $w(x)|x-u|^{-1} \in L^{2}$. Therefore $v_{u} w \in L^{2}$ and, by Theorem 4.4, there is a unique solution $f_{u}$ to Eq. (4.4), with this $v_{u}$, satisfying $w f_{u} \in L^{2}$. Moreover by Lemma 4.6 and the properties of $v_{u}, f_{u}$ [defined by the right side of (4.4)] is continuous away from $u$ and goes to zero at infinity. (i) Let $S=\{x \mid f(x)<0\}$. Since $f_{u} \rightarrow \infty$ as $x \rightarrow u, S$ is disjoint from $u$ and open (since $f_{u}$ is continuous away from $u$ ). On $S$, the distributional laplacian of $f_{u}$ is given by

$$
-(4 \pi)^{-1} \Delta f_{u}=-w^{2} f_{u}-(4 \pi)^{-1} \Delta V \geqq-w^{2} f_{u} \geqq 0 .
$$

Then (i) follows from MMP. (ii) Call $\psi=f_{2}-f_{1} . \psi$ is continuous everywhere and goes to zero at infinity. Let $S=\{x \mid \psi(x)<0\}$. $S$ is open and $\psi=0$ on $\partial S \cup\{\infty\}$. On $S$, $-(4 \pi)^{-1} \Delta \psi=w_{1}^{2} f_{1}-w_{2}^{2} f_{2} \geqq-w_{2}^{2} \psi>0$ and (ii) follows using MMP. (iii) is a consequence of (i) and the linearity of $f_{u}$ in $v_{u}$.

Theorem 4.9. (Asymptotic Behavior of $f(x)$ ). Consider $v(x)=|x|^{-1}$ and $w$ as in Lemma 4.8 and, moreover, $w(x)^{2} \geqq c|x|^{-2}$ for $|x|>R$ and some $c>0$. Then $f(x)$ $\leqq M(c)|x|^{-\alpha(c)}$ for $|x|>R$ where $\alpha(c)=(1+\sqrt{1+16 \pi c}) / 2$ and $M(c)=\alpha(c)^{-1} R^{\alpha(c)-1}$. 
Proof. Take $w_{1}$ defined by $w_{1}(x)^{2}=c|x|^{-2}$ for $|x|>R$ and $w_{1}^{2}=0$ for $|x|<R$, the solution to (4.4) corresponding to this $w$ is given by $f_{1}(x)=M(c)|x|^{-\alpha(c)}$ for $|x|>R$ and $f_{1}(x)=|x|^{-1}(1+a|x|)$ for $|x|<R$ with $M(c)=\alpha(c)^{-1} R^{\alpha(c)-1}$ and $a=(1-\alpha(c)) /(\alpha(c) R)$. We have $w(x)^{2} \geqq w_{1}(x)^{2}$ all $x$ and hence, by Lemma 4.8(ii) $f(x)$ $\leqq f_{1}(x)$. In particular $f(x) \leqq M(c)|x|^{-\alpha(c)}$ for $|x|>R$.

To close this section we prove the following,

Lemma 4.10. If $v(x)=|x|^{-1}$, and $w$ as in Lemma 4.8, then $\int w(x)^{2} f(x) d x \leqq 1$, If, moreover, $w(x)^{2} \geqq c|x|^{-2}$ for $|x|>R$ (for some $c, R$ ), then $\int w(x)^{2} f(x) d x=1$.

Proof. Assume $\int w^{2} f>1$. Define the spherical average $[f](r) \equiv(4 \pi)^{-1} \int_{S_{2}} f(r \Omega) d \Omega$. From (4.4) we have $[f](r)=r^{-1}-\int w(y)^{2} f(y)\left[(4 \pi)^{-1} \int_{S_{2}} d \Omega|r \Omega-y|^{-1}\right] d y$. Using the well known formula $(4 \pi)^{-1} \int_{S_{2}} d \Omega|r \Omega-y|^{-1}=\{\max (r,|y|)\}^{-1}$ we get $[f](r)$ $\leqq r^{-1}\left(1-\int_{\mid y / \leqq r} w(y)^{2} f(y) d y\right)$. Therefore for $r$ large enough $[f](r)<0$ which contradicts Lemma 4.8(i). Hence $\int w^{2} f \leqq 1$. Let us now consider $w$ such that $w(x)^{2}$ $\geqq c|x|^{-2}$ for $|x|>R$. [f] $(r) \geqq r^{-1}\left(1-\int w^{2} f\right)$ by the same arguments as above. If $\int w^{2} f<1$, then $[f](r) \geqq d r^{-1}$ for some positive $d$ which contradicts Theorem 4.9 .

\section{IV.2. Proof of Theorem 2.6: Properties of the TF Potential}

The strategy to prove that $\underline{z} \rightarrow \phi(\underline{z}, x) \in C^{1}$ is the following: we first show that a unique solution to Eq. (4.2) exists (Lemma 4.11) and is continuous in $\underline{z}$ uniformly with respect to $x$ (Lemma 4.13). We then show that $\phi_{i}^{\varepsilon}(\underline{z}, x) \equiv \varepsilon^{-1}\left[\phi\left(\underline{z}+\varepsilon \underline{e}_{i}, x\right)\right.$ $-\phi(\underline{z}, x)]$, with $\underline{e}_{i}=\left(\delta_{i}^{i}\right)$ a unit vector in $\mathbb{R}_{+}^{k}$ along $z_{i}$, converges to $\phi_{i}(\underline{z}, x)$ as $\varepsilon \rightarrow 0$ uniformly in $x$. (Lemma 4.14). We then imitate the same argument to show that $\phi \in C^{2}$.

In what follows we study the equation

$$
\phi_{u}(\underline{z}, x)=|x-u|^{-1}-(3 / 2) \int d y|x-y|^{-1} \phi(\underline{z}, y)^{1 / 2} \phi_{u}(z, y) \text {. }
$$

Note that $w \equiv(3 / 2)^{1 / 2} \phi^{1 / 4} \in L_{w}^{3}$ (since $w$ goes as $|x|^{-1}$ at infinity) and $w \in L^{p}$ for all $p \in(3,12)[(\mathrm{P}-4),(\mathrm{P}-6)]$. In particular $w \in L^{6-\varepsilon} \cap L^{6+\varepsilon}$, for some $\in>0$, therefore $|x-u|^{-1} w \in L^{2}$ as discussed in the proof of Lemma 4.8.

Lemma 4.11. (Existence of $\phi_{u}(\underline{z}, x)$ ). There is a unique $\phi_{u}(\underline{z}, x)$ satisfying Eq. (4.9) with $\phi_{u} \phi^{1 / 4} \in L^{2}$, and it has the following properties:

(i) $\phi_{u}(z, x)-|x-u|^{-1}$ is a bounded continuous function going to zero at infinity.

(ii) $\underline{z} \rightarrow \phi_{u}(z, x)$ is non-negative and decreasing

(iii) $\underline{z} \rightarrow \phi_{u}(z, x)$ is ray-convex.

(iv) For every $\alpha<(1+\sqrt{73}) / 2 \simeq 4.77$, there exists an $R(\alpha)<\infty$ and a finite number $M(\alpha)$ such that $\phi_{u}(\underline{z}, x) \leqq M(\alpha)|x|^{-\alpha}$ for $|x| \geqq R(\alpha)$.

Proof. Since $w \in L_{w}^{3}$ and $|x-u|^{-1} w \in L^{2}$, Theorem 4.4 implies the existence of a unique $\phi_{u}(z, x)$ satisfying (4.9) with $w \phi_{u} \in L^{2}$. (i) follows from Lemma 4.7, since 


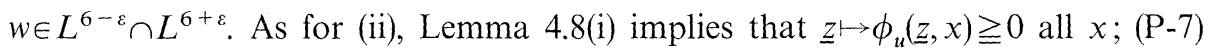
together with Lemma 4.8(ii) imply that $\underline{z} \mapsto \phi_{u}(\underline{z}, x)$ is decreasing. To prove (iii), let $\underline{z}_{1}, \underline{z}_{2} \in \mathbb{R}_{+}^{k}$ with $\underline{z}_{1}-\underline{z}_{2} \in \mathbb{R}_{+}^{k}$ and define $\underline{z}=\lambda \underline{z}_{1}+(1-\lambda) \underline{z}_{2}$ with $0 \leqq \lambda \leqq 1$. Define $\psi(x) \equiv \lambda \phi_{u}\left(\underline{z}_{1}, x\right)+(1-\lambda) \phi_{u}\left(\underline{z}_{2}, x\right)-\phi_{u}(\underline{z}, x)$. Because of (i) $\psi(x)$ is continuous everywhere and goes to zero at infinity. Then $S=\{x \mid \psi(x)<0\}$ is open and $\psi=0$ on $\partial S \cup\{\infty\}$. From (4.9)

$$
\begin{aligned}
-(4 \pi)^{-1} \Delta \psi= & \frac{3}{2}\left\{-\lambda \phi\left(\underline{z}_{1}, x\right)^{1 / 2} \phi_{u}\left(\underline{z}_{1}, x\right)-(1-\lambda) \phi\left(\underline{z}_{2}, x\right)^{1 / 2} \phi_{u}\left(\underline{z}_{2}, x\right)\right. \\
& \left.+\phi(\underline{z}, x)^{1 / 2} \phi_{u}(\underline{z}, x)\right\} .
\end{aligned}
$$

Because of (P-9), (P-7) and part (ii) (since $\underline{z}_{1}-\underline{z}_{2} \in \mathbb{R}_{+}^{k}$ ) we have $-(4 \pi)^{-1} \Delta \psi \geqq 0$. Hence (iii) follows using MMP. (iv) given $\alpha<(1+\sqrt{73}) / 2$ (i.e. $c<(9 / 2 \pi)$ ) there exists $R(c)<\infty$ such that $w(x)^{2} \equiv(3 / 2) \phi(z, x)^{1 / 2} \geqq c|x|^{-4}(\mathrm{P}-4)$. Hence, by Theorem 4.9, $\phi_{u}(\underline{z}, x) \leqq M(\alpha)|x|^{-\alpha}$ for $|x| \geqq R(c)$.

Remark. In the atomic case, Hille [7] used methods of ordinary differential equations to prove that the asymptotic formula with $\alpha=(1+\sqrt{73}) / 2$ was exact $[[7]$, Eq. (4.5)].

We now prove a general theorem that we will later need:

Theorem 4.12. Let $f$ be a real (or complex) function on $\mathbb{R}_{+}^{k}$. Suppose $f$ satisfies the following condition:

$$
\left|f\left(z_{1}\right)-f\left(\underline{z}_{2}\right)\right|<K\left\|\underline{z}_{1}-\underline{z}_{2}\right\|_{2}^{\alpha}
$$

for all $\underline{z}_{1}, \underline{z}_{2} \in \mathbb{R}_{+}^{k}$ such that $\underline{z}_{1}-\underline{z}_{2} \in \mathbb{R}_{+}^{k}$, for some $\alpha>0$ and some $K>0$, then $\underline{z} \mapsto f(\underline{z})$ is continuous in the whole of $\mathbb{R}_{+}^{k}$.

Proof. Assume first that $\underline{z} \in \operatorname{Int}\left(\mathbb{R}_{+}^{k}\right)$. Let $\underline{n}=(1,1, \ldots, 1)$, and $\underline{z}_{0} \equiv \underline{z}-\delta \underline{n}$, with $\delta \leqq \min _{1 \leqq i \leqq k}\left(z_{i}\right)$ (i.e. $\underline{z}_{0} \in \mathbb{R}_{+}^{k}$ ). Let $\underline{z}^{\prime} \in B(\underline{z}, \delta)$, the ball of radius $\delta$ centered at $\underline{z}$. Applying (4.10) twice we get $\left|f\left(\underline{z}^{\prime}\right)-f(\underline{z})\right| \leqq\left(\left\|\underline{z}^{\prime}-\underline{z}_{0}\right\|_{2}^{\alpha}+\left\|\underline{z}-\underline{z}_{0}\right\|_{2}^{\alpha}\right) K$, because $\underline{z}^{\prime}-\underline{z}_{0} \in \mathbb{R}_{+}^{k}, \underline{z}-\underline{z}_{0} \in \mathbb{R}_{+}^{k}$. But, as $\delta \rightarrow 0,\left\|\underline{z}-\underline{z}_{0}\right\|_{2} \rightarrow 0$ and $\left\|\underline{z}^{\prime}-\underline{z}_{0}\right\| \rightarrow 0$ uniformly in $B(\underline{z}, \delta)$, so $f$ is continuous at $\underline{z}$. Now, if $\underline{z}$ is in one face, $F$, of $\mathbb{R}_{+}^{k}$ (of dimension $0 \leqq l$ $<k$ ) the same argument can be repeated using $\underline{\tilde{n}} \equiv$ projection of $\underline{n}$ on $F$.

Lemma 4.13. (Continuity of $\phi_{u}(z, x)$ in $\left.\underline{z}\right)$. $\phi_{u}(z, x)$ defined as the solution to (4.9) (Satisfying $\phi^{1 / 4} \phi_{u} \in L^{2}$ ) is continuous for all $\underline{z} \in \mathbb{R}_{+}^{k}$ uniformly with respect to $x$.

Proof. We divide the proof into two steps. First we prove continuity at $\underline{z} \neq \underline{0}$, and then at $\underline{z}=\underline{0}$. (i) $\underline{z} \neq \underline{0}$. There is a $\underline{z}^{*} \in \mathbb{R}_{+}^{k}, \underline{z}^{*} \neq \underline{0}$ such that $\underline{z}-\underline{z}^{*} \in \mathbb{R}_{+}^{k}$. Let $\underline{z}_{1}$, $\underline{z}_{2} \in\left(\underline{z}^{*}+\mathbb{R}_{+}^{k}\right)$ with $\underline{z}_{1}-\underline{z}_{2} \in \mathbb{R}_{+}^{k}$. From Eq. (4.9) we get,

$$
\begin{aligned}
\phi_{u}\left(\underline{z}_{2}, x\right)-\phi_{u}\left(\underline{z}_{1}, x\right)= & (3 / 2) \int|x-y|^{-1} \phi_{u}\left(\underline{z}_{1}, y\right)\left[\phi\left(\underline{z}_{1}, y\right)^{1 / 2}-\phi\left(\underline{z}_{2}, y\right)^{1 / 2}\right] d y \\
& +(3 / 2) \int|x-y|^{-1} \phi\left(\underline{z}_{2}, y\right)^{1 / 2}\left[\phi_{u}\left(\underline{z}_{1}, y\right)-\phi_{u}\left(\underline{z}_{2}, y\right)\right] .
\end{aligned}
$$

Since $\underline{z}_{1}-\underline{z}_{2} \in \mathbb{R}_{+}^{k}, \phi_{u}\left(\underline{z}_{2}, x\right)-\phi_{u}\left(\underline{z}_{1}, x\right) \geqq 0$ because of Lemma 4.11(ii). Hence, (4.11) implies

$$
\left|\phi_{u}\left(\underline{z}_{2}, x\right)-\phi_{u}\left(\underline{z}_{1}, x\right)\right|<(3 / 2) \int|x-y|^{-1} \phi_{u}\left(\underline{z}^{*}, y\right)\left(\phi\left(\underline{z}_{1}, y\right)^{1 / 2}-\phi\left(\underline{z}_{2}, y\right)^{1 / 2}\right) .
$$


Because of (P-8) we have,

$$
\phi\left(\underline{z}_{1}, y\right)^{1 / 2}-\phi\left(\underline{z}_{2}, y\right)^{1 / 2} \leqq \phi\left(\underline{z}_{1}-\underline{z}_{2}, y\right)^{1 / 2} \leqq\left\|\underline{z}_{1}-\underline{z}_{2}\right\|_{2}^{1 / 2} \sum_{i=1}^{k} \frac{1}{\left|y-R_{i}\right|^{1 / 2}},
$$

where the last inequality follows from Eq. (4.1). Hence

$$
\left|\phi_{u}\left(\underline{z}_{2}, x\right)-\phi_{u}\left(\underline{z}_{1}, x\right)\right|<\left\|\underline{z}_{1}-\underline{z}_{2}\right\|_{2}^{1 / 2} g(x),
$$

where

$$
g(x)=(3 / 2) \int|x-y|^{-1} \sum_{i=1}^{k}\left(\frac{1}{\left|y-R_{i}\right|^{1 / 2}}\right) \phi_{u}\left(z^{*}, y\right) .
$$

By Young's inequality $g(x) \in L^{\infty} \quad$ because $\quad|x|^{-1} \in L^{4}+L^{5 / 2} \quad$ and $\left|y-R_{i}\right|^{-1 / 2} \phi_{u}\left(z^{*}, y\right) \in L^{p}$ for any $1 \leqq p<2$, in particular for $p=4 / 3$ and $p=5 / 3$. [Lemma 4.11(i), (iv)]. Theorem 4.12 and Eq. (4.13) then imply that $\phi_{u}(z, x)$ is continuous in $\underline{z}$, uniformly with respect to $x$, for all $\underline{z} \in\left(\underline{z}^{*}+\mathbb{R}_{+}^{k}\right)$. But $\bigcup_{z^{*}}\left(\underline{z}^{*}+\mathbb{R}_{+}^{k}\right)$ $=\mathbb{R}_{+}^{k} \backslash\{\underline{0}\}$. (ii) $\underline{z}=\underline{0}$, Equation (4.9) and Lemma 4.11(ii) imply

$$
\left|\phi_{u}(\underline{z}, x)-\phi_{u}(\underline{0}, x)\right| \leqq h(x) \equiv \int|x-y|^{-1} \phi(\underline{z}, y)^{1 / 2}|y-u|^{-1} d y .
$$

Using Young's inequality we get,

$$
\begin{gathered}
\|h(x)\|_{\infty} \leqq \\
+\left\|\phi(\underline{z}, \cdot)^{1 / 4}\right\|_{6}\left(\left\||x|_{<}^{-1}\right\|_{42 / 17}\left\|\phi^{1 / 4}|y-u|^{-1}\right\|_{7 / 3}\right. \\
\left.+\left\||x|_{>}^{-1}\right\|_{4}\left\|\phi^{1 / 4}|y-u|^{-1}\right\|_{12 / 7}\right)
\end{gathered}
$$

and thus $\|h(x)\|_{\infty} \leqq c\left\|\phi^{1 / 4}\right\|_{6}$, with $c<\infty$ because $\phi^{1 / 4}|y-u|^{-1} \in L^{p}$ for any $(3 / 2)<p$ $<(12 / 5)$ and $|x|_{<}^{-1} \in L^{p}(p<3),|x|_{>}^{-1} \in L^{p}(p>3)$. From (4.14) we finally get $\| \phi_{u}(\underline{z}, x)$ $-\phi_{u}(\underline{0}, x)\left\|_{\infty} \leqq c\right\| \phi^{1 / 4} \|_{6}=c\left(\int \varrho\right)^{1 / 6}=c z^{1 / 6}$, where $z=\sum_{i=1}^{k} z_{i}$.

Let us define $\phi_{i}(\underline{z}, x)$ to be $\phi_{u}(\underline{z}, x)$ with $u=R_{i}$. Then the last step to prove that $\phi(\underline{z}, x) \in C^{1}\left(\mathbb{R}_{+}^{k}\right)$, uniformly with respect to $x$, is the following:

Lemma 4.14. (Convergence of $\phi_{i}^{\varepsilon}(\underline{z}, x)$ to $\left.\phi_{i}(\underline{z}, x)\right)$. Let $\phi_{i}^{\varepsilon}(\underline{z}, x) \equiv \varepsilon^{-1}\left[\phi\left(\underline{z}+\varepsilon \underline{e}_{i}, x\right)\right.$ $-\phi(\underline{z}, x)]$ with $\underline{e}_{i}=\left(\delta_{i}^{i}\right)$ being a unit vector in $\mathbb{R}_{+}^{k}$, along $z_{i}$ and $\varepsilon \geqq-z_{i}$. Then $\phi_{i}^{\varepsilon}(\underline{z}$, $x) \rightarrow \phi_{i}(z, x)$ as $\varepsilon \rightarrow 0$, uniformly with respect to $x$.

Proof. (i) Consider first $\varepsilon>0$. We will prove the following,

$$
\begin{aligned}
& \phi_{i}^{\varepsilon}(\underline{z}, x)-\phi_{i}\left(\underline{z}+\varepsilon \underline{e}_{i}, x\right) \geqq 0, \\
& \phi_{i}^{\varepsilon}(\underline{z}, x)-\phi_{i}(\underline{z}, x) \leqq 0 .
\end{aligned}
$$

Consider $\psi(x) \equiv \phi_{i}^{\varepsilon}(\underline{z}, x)-\phi_{i}\left(\underline{z}+\varepsilon_{i}, x\right)$. By Lemma 4.11 and $(\mathrm{P}-2), \psi$ is continuous for all $x$ and goes to zero at infinity. Then $S \equiv\{x \mid \psi<0\}$ is open and $\psi=0$ on $\partial S \cup\{\infty\}$. On $S$,

$$
\begin{aligned}
-(4 \pi)^{-1} \Delta \psi= & \varepsilon^{-1}\left[\phi(\underline{z}, x)^{3 / 2}-\phi\left(\underline{z}+\varepsilon \underline{e}_{i}, x\right)^{3 / 2}\right] \\
& +(3 / 2) \phi_{i}\left(\underline{z}+\varepsilon \underline{e}_{i}, x\right) \cdot \phi\left(z+\varepsilon \underline{e}_{i}, x\right)^{1 / 2} \\
& \geqq(2 \varepsilon)^{-1} \phi\left(\underline{z}+\varepsilon \underline{e}_{i}, x\right)^{1 / 2} \phi(\underline{z}, x)\left[\mu^{2}-3+2 \mu^{-1}\right],
\end{aligned}
$$


where $\mu^{2} \equiv \phi\left(\underline{z}+\varepsilon \underline{e}_{i}, x\right) / \phi(\underline{z}, x) \geqq 1\left[\right.$ by (P-7)]. Hence $\mu^{2}-3+2 \mu^{-1} \geqq 0$. MMP then implies (4.15a). The proof of (4.15b) is analogous. From (4.15) and Lemma 4.13, $\left\|\phi_{i}^{\varepsilon}(z, x)-\phi_{i}(\underline{z}, x)\right\|_{\infty} \rightarrow 0$ as $\varepsilon \downarrow 0$. (ii) If $-z_{i}<\varepsilon<0,(4.15 \mathrm{a}, \mathrm{b})$ imply

$$
\phi_{i}(\underline{z}, x) \leqq \phi_{i}^{\varepsilon}(z, x) \leqq \phi_{i}\left(\underline{z}+\varepsilon \underline{e}_{i}, x\right)
$$

which in turn implies $\left\|\phi_{i}^{\varepsilon}(z, x)-\phi_{i}(\underline{z}, x)\right\|_{\infty} \rightarrow 0$ as $\varepsilon \uparrow 0$.

If we denote by $\phi_{i j}(\underline{z}, x)$ the derivative of $\phi_{i}(\underline{z}, x)$ with respect to $z_{j}$, we formally get (from (4.2)) :

$$
\begin{aligned}
-\phi_{i j}(z, x)= & (3 / 4) \int|x-y|^{-1} \phi(z, y)^{-1 / 2} \phi_{i}(z, y) \phi_{j}(z, y) d y \\
& \left.-(3 / 2) \int \mid x-y\right)^{-1} \phi(\underline{z}, y)^{1 / 2}\left(-\phi_{i j}(\underline{z}, y)\right) d y .
\end{aligned}
$$

As we have already mentioned, the strategy to prove that $\phi(\underline{z}, x)$ is in $C^{2}\left(\mathbb{R}_{+}^{k} \backslash \underline{0}\right)$, uniformly with respect to $x$, will be the same as before. Now there will be an additional difficulty, namely the control of $\phi(z, y)^{-1 / 2}$. Let us start proving that a solution to (4.17) indeed exists.

Lemma 4.15. (Existence of $\phi_{i j}(\underline{z}, x)$ ). For $\underline{z} \neq \underline{0}$, there is a unique $\phi_{i j}(\underline{z}, x)$ satisfying Eq. (4.17) and such that $\phi_{i j} \phi^{1 / 4} \in L^{2}$. Moreover:

(i) $\phi_{i j}(z, x)$ is continuous for all $x$. It is bounded and goes to zero at infinity.

(ii) $-\phi_{i j}(z, x)$ is non-negative and so is $\sum_{1 \leqq i, j \leqq k} \bar{c}_{i}\left(-\phi_{i j}(z, x)\right) c_{j}$, and $\underline{c} \in C^{k}$.

(iii) $-\phi_{i j}(z, x)$ is a decreasing function of $\underline{z}$.

Proof. Note first that, for $\underline{z} \neq \underline{0}, \phi(\underline{z}, \cdot)^{-1 / 4} \phi_{i}(\underline{z}, \cdot) \in L^{q}$ for any $1 \leqq q<4$. In fact, for $\underline{z} \neq \underline{0}, \phi$ is strictly positive $(\mathrm{P}-3)$ and $\phi(\underline{z}, x) \geqq \tilde{c}|x|^{-4}$ for $|x|>R \equiv 2 \max \left|R_{i}\right|$ and some positive constant $\tilde{c}$ (P-4). Because of Lemma 4.11(iv), $\phi_{i} \phi^{-1 / 4} \leqq c_{1}|x|^{1-\alpha}$ for $|x|$ $>R(\alpha)$ with $4<\alpha<4.77$. Then if $B(0, R(\alpha))=\{x \| x \mid \leqq R(\alpha)\}, \phi_{i} \phi^{-1 / 4} \in L^{p}\left(\mathbb{R}^{3} \backslash B(0\right.$, $R(\alpha))), \forall p \geqq 1$. Inside $B(0, R(\alpha))$ and away from $R_{i} \phi^{-1 / 4} \phi_{i}$ is bounded since $B$ is compact, (P-4) and Lemma 4.11(i). In a neighborhood of $R_{i} \phi_{i} \phi^{-1 / 4}$ behaves like $\left|x-R_{i}\right|^{-3 / 4}$, hence $\phi_{i} \phi^{-1 / 4} \in L^{q}$ for any $1 \leqq q<4$. Therefore, $\phi_{i} \phi^{-1 / 4} \phi_{j} \phi^{-1 / 4} \in L^{s}$ for $1 \leqq s<2$ and, since $|x|^{-1} \in L_{w}^{3}, v_{i j} \equiv(3 / 4)|x|^{-1} *\left(\phi_{i} \phi^{-1 / 4} \phi_{j} \phi^{-1 / 4}\right) \in L^{t}$ for any $3<t$ $<\infty$ (by the generalized Young's inequality). Moreover, $v_{i j} \phi^{1 / 4} \in L^{2}$ because $\phi^{1 / 4} \in L^{p}, 3<p<12$. Finally, since $\phi^{1 / 4} \in L_{w}^{3}$, Theorem 4.4 implies that there is a unique solution $\phi_{i j}(\underline{z}, x)(\underline{z} \neq \underline{0})$ to Eq. (4.17) satisfying $\phi^{1 / 4} \phi_{i j} \in L^{2}$. (i) By Lemma 4.7, $\phi_{i j}+v_{i j}$ is a bounded continuous function going to zero at infinity. Lemma 4.6 shows that $v_{i j}$ is continuous, bounded and goes to zero at infinity because $\left(\phi^{-1 / 4} \phi_{i}\right)\left(\phi^{-1 / 4} \phi_{j}\right) \in L^{s}$ for any $1 \leqq s<2$ and $|x|^{-1} \in L^{4}+L^{5 / 2}$. (ii) Follows from Lemma 4.8 because $v_{i j}$ is superharmonic and so is $\sum \bar{c}_{i} v_{i j} c_{j}$. Lemma 4.11(ii) and (P-7) imply that $v_{i j}\left(\underline{z}_{1}, x\right)-v_{i j}\left(\underline{z}_{2}, x\right)$ is superharmonic if $\underline{z}_{1}-\underline{z}_{2} \in \mathbb{R}_{+}^{k}$. Then (iii) follows from Lemma 4.8(ii) and (iii).

In order to prove the asymptotic behavior of $\phi_{i j}(\underline{z}, x)$ we will need the following comparison Lemma. See also [[1], Theorem 4.7].

Lemma 4.16. Assume that $f_{1}, f_{2}$ are continuous positive functions on $\{x|| x \mid \geqq R\}$ with the following properties:

(i) $f_{1}(x), f_{2}(x) \rightarrow 0$ as $|x| \rightarrow \infty$. 
(ii) $-(4 \pi)^{-1} \Delta f_{1}=\varrho_{1}-w_{1} f_{1},-(4 \pi)^{-1} \Delta f_{1}=\varrho_{2}-w_{2} f_{2}$, where the derivatives and equalities are in distributional sense, and $\varrho_{1}(x) \geqq \varrho_{2}(x), 0 \leqq w_{1}(x) \leqq w_{2}(x)$. $|x| \geqq R$.

(iii) $f_{1}(x) \geqq f_{2}(x)$ for all $x$ such that $|x|=R$. Then $f_{1}(x) \geqq f_{2}(x)$ for all $x$ such that

Proof. Define $\psi=f_{1}-f_{2}$. Let $S=\{x \mid \psi(x)<0\}$, which is open. On $S-(4 \pi)^{-1} \Delta \psi$ $=\left(\varrho_{1}-\varrho_{2}\right)-\mathrm{w}_{1} \psi+\mathrm{f}_{2}\left(\mathrm{w}_{2}-\mathrm{w}_{1}\right) \geqq 0$. The Lemma follows from MMP since, by (i) and (iii), $\psi=0$ at $\infty$ and $\psi>0$ on $\partial S$.

Lemma 4.17. (Asymptotic Behavior of $\phi_{i j}(z, x)$ ). Let $\alpha<(1+\sqrt{73}) / 2 \simeq 4.77$ and let $\phi_{i j}(z, x)$ be the solution to (4.17) satisfying $\phi^{1 / 4} \phi_{i j} \in L^{2}$. Then there exists an $R(\alpha)<\infty$ such that $-\phi_{i j}(z, x) \leqq B(\alpha)|x|^{-\alpha}$ for some $B(\alpha)>0$, when $|x|>R(\alpha)$.

Remark. The remark below Lemma 4.11 also applies to $-\phi_{i j}$.

Proof. Consider the equation,

$$
-(4 \pi)^{-1} \Delta f=b r^{2(1-\alpha)}-d r^{-2} f, \text { for } r=|x|>R
$$

where $\alpha(\alpha-1)=4 \pi d$, together with the boundary condition $f(x)=N$ for $|x|=R$. The solution $f(x)$ to $(4.18)$, going to zero at infinity is,

$$
f(x)=N(R / r)^{\alpha}+4 \pi b(3 \alpha-5)^{-1}(\alpha-4)^{-1} r^{-\alpha} R^{4-\alpha}\left(1-(R / r)^{\alpha-4}\right) .
$$

Given any $\alpha(c)<(1+\sqrt{73}) / 2$ there exists $R(c)<\infty$ such that $w(x)^{2} \equiv(3 / 2) \phi(z, x)^{1 / 2}$ $\geqq c|x|^{-4}(\mathrm{P}-4)$ and $\phi_{i}(\underline{z}, x) \leqq M(\alpha(c))|x|^{-\alpha(c)}$ (Lemma 4.11(iv)) for $|x| \geqq R(c)$. Hence $(3 / 4) \phi^{-1 / 2} \phi_{i} \phi_{j} \leqq(3 / 4) M(c)^{2} c^{-1}|x|^{2-2 \alpha(c)}$ for $|x| \geqq R(c)$. Using (4.17), (4.18) and the comparison Lemma we get:

$$
-\phi_{i j}(z, x) \leqq\left[3 \pi M^{2} c^{-1}(3 \alpha-5)^{-1}(\alpha-4)^{-1}\right] r^{-\alpha} R^{4-\alpha}\left(1-(R / r)^{\alpha-4}\right)+N(R / r)^{\alpha}
$$

for $|x| \geqq R$, with $N=\max _{|x|=R}\left(-\phi_{i j}(z, x)\right)$ which is finite because $\phi_{i j}$ is bounded (Lemma 4.15(i)).

Lemma 4.18. (Continuity of $\phi_{i j}(\underline{z}, x)$ ). $\phi_{i j}(\underline{z}, x)$ is continuous in $\underline{z}$ for all $\underline{z} \in \mathbb{R}_{+}^{k} \backslash\{\underline{0}\}$, uniformly in $x$.

Proof. Let $\underline{z}^{*} \in \mathbb{R}_{+}^{k} \backslash\{\underline{0}\}$ such that $\underline{z}-\underline{z}^{*} \in \mathbb{R}_{+}^{k}$. Let $\underline{z}_{1}, \underline{z}_{2} \in\left(\underline{z}^{*}+\mathbb{R}_{+}^{k}\right)$ with $\left(\underline{z}_{1}-\underline{z}_{2}\right) \in \mathbb{R}_{+}^{k}$. Lemma 4.15(iii) and Eq. (4.17) imply

$$
0 \leqq \phi_{i j}\left(\underline{z}_{1}, x\right)-\phi_{i j}\left(\underline{z}_{2}, x\right) \leqq I(x)+J(x)
$$

with

$$
\begin{aligned}
I(x)= & (3 / 4) \int|x-y|^{-1}\left\{\phi\left(\underline{z}_{2}, y\right)^{-1 / 2} \phi_{i}\left(\underline{z}_{2}, y\right) \phi_{j}\left(\underline{z}_{2}, y\right)\right. \\
& \left.-\phi\left(\underline{z}_{1}, y\right)^{-1 / 2} \phi_{i}\left(\underline{z}_{1}, y\right) \phi_{j}\left(\underline{z}_{1}, y\right)\right\} d y
\end{aligned}
$$

and

$$
J(x)=(3 / 2) \int|x-y|^{-1}\left(-\phi_{i j}\left(z^{*}, y\right)\right)\left[\phi\left(\underline{z}_{1}, y\right)^{1 / 2}-\phi\left(\underline{z}_{2}, y\right)^{1 / 2}\right] d y .
$$

To estimate $J(x)$ we use (4.12) to get.

$$
J(x) \leqq\left\|\underline{z}_{1}-\underline{z}_{2}\right\|_{2}^{1 / 2} g_{1}(x),
$$


where $g_{1}(x)=(3 / 2) \int|x-y|^{-1}\left(-\phi_{i j}\left(z^{*}, y\right)\right) \sum_{n=1}^{k}\left|y-R_{n}\right|^{-1 / 2} d y$. Since for $\underline{z} \neq \underline{0} \phi_{i j}$ is bounded everywhere [Lemma 4.15(i)] and since it goes to zero at least as fast as $r^{-4.5}$ at infinity (Lemma 4.17), $|y|^{-1 / 2} \phi_{i j} \in L^{p}$ for any $1 \leqq p<6$. In particular $\left|y-R_{n}\right|^{-1 / 2} \phi_{i j} \in L^{4 / 3} \cap L^{2}$ and therefore, by Young's inequality $g_{1} \in L^{\infty}$ because $|x|^{-1} \in L^{4}+L^{2} . I(x)$ is decomposed as,

$$
I(x)=I_{1}(x)+I_{2}(x)+I_{3}(x)
$$

where

$$
\begin{aligned}
I_{1}(x)= & (3 / 4) \int|x-y|^{-1}\left(\phi\left(\underline{z}_{2}, y\right)^{-1 / 2}-\phi\left(\underline{z}_{1}, y\right)^{-1 / 2}\right) \phi_{i}\left(\underline{z}_{2}, y\right) \phi_{j}\left(\underline{z}_{2}, y\right) d y, \\
I_{2}(x)= & (3 / 4) \int|x-y|^{-1}\left(\phi_{i}\left(\underline{z}_{2}, y\right)\right. \\
& \left.-\phi_{i}\left(\underline{z}_{1}, y\right)\right) \phi\left(\underline{z}_{1}, y\right)^{-1 / 2} \phi_{j}\left(\underline{z}_{2}, y\right) d y, \\
I_{3}(x)= & (3 / 4) \int \mid\left(x-\left.y\right|^{-1}\left(\phi_{j}\left(\underline{z}_{2}, y\right)\right.\right. \\
& \left.-\phi_{j}\left(\underline{z}_{1}, y\right)\right) \phi\left(\underline{z}_{1}, y\right)^{-1 / 2} \phi_{i}\left(\underline{z}_{1}, y\right) d y .
\end{aligned}
$$

To find a bound for $I_{1}(x)$ we use Lemma 4.11(ii) and the following estimate:

$$
\begin{aligned}
& \phi\left(\underline{z}_{2}, y\right)^{-1 / 2}-\phi\left(\underline{z}_{1}, y\right)^{-1 / 2} \\
& \quad=\left[\phi\left(\underline{z}_{1}, y\right)-\phi\left(\underline{z}_{2}, y\right)\right] \phi\left(\underline{z}_{1}, y\right)^{-1} \phi\left(\underline{z}_{2}, y\right)^{-1}\left[\phi\left(\underline{z}_{1}, y\right)^{-1 / 2}+\phi\left(\underline{z}_{2}, y\right)^{-1 / 2}\right]^{-1} \\
& \quad \leqq\left\|\underline{z}_{1}-\underline{z}_{2}\right\|_{2}\left[\sum_{i=1}^{k}\left|x-R_{i}\right|^{-1}\right](1 / 2) \phi\left(\underline{z}^{*}, y\right)^{-3 / 2} .
\end{aligned}
$$

which follows from Eq. (4.1) and (P-7). Hence we have

$$
I_{1}(x) \leqq\left\|\underline{z}_{1}-\underline{z}_{2}\right\|_{2} g_{2}(x)
$$

with $\quad g_{2}(x)=(3 / 8) \int|x-y|^{-1} \phi\left(z^{*}, y\right)^{-3 / 2} \phi_{i}\left(z^{*}, y\right) \phi_{j}\left(z^{*}, y\right) \sum_{n=1}^{k}\left|x-R_{n}\right|^{-1} d y$. Since $\phi_{i} \phi^{-1} \in L^{p}$ for any $p \geqq 6,\left(\phi_{i} \phi^{-1}\right)\left(\phi_{j} \phi^{-1}\right) \in L^{p}, p \geqq 3$. Also, $\phi^{1 / 2} \sum_{n=1}^{k}\left|x-R_{n}\right|^{-1} \in L^{q}$, $1<q<2$ and therefore $\phi^{-3 / 2} \phi_{i} \phi_{j} \sum_{n=1}^{k}\left|x-R_{n}\right|^{-1} \in L^{s}, 1 \leqq s<2$. Since $|x|^{-1} \in L^{4}$ $+L^{5 / 2} g_{2} \in L^{\infty}$ by Young's inequality. Equations (4.12) and (4.23) and Lemma (4.13) imply

$$
I_{2}(x) \leqq(3 / 4) \int|x-y|^{-1} g(y)\left\|\underline{z}_{1}-\underline{z}_{2}\right\|_{2}^{1 / 2} \phi\left(z^{*}, y\right)^{-1 / 2} \phi_{j}\left(z^{*}, y\right) d y
$$

with

$$
g(x)=(3 / 2) \int|x-y|^{-1} \sum_{i=1}^{k}\left|y-R_{i}\right|^{-1 / 2} \phi_{i}\left(z^{*}, y\right) d y \in L^{\infty}
$$

because of Lemma 4.13. Then

$$
I_{2}(x) \leqq\left\|\underline{z}_{1}-\underline{z}_{2}\right\|_{2}^{1 / 2} g_{3}(x)
$$

with

$$
g_{3}(x)=(3 / 4)\|g\|_{\infty} \int|x-y|^{-1} \phi\left(z^{*}, y\right)^{-1 / 2} \phi_{j}\left(z^{*}, y\right) d y .
$$


Since $\phi^{-1 / 2} \phi_{j} \in L^{p}$ for any $(3 / 2.5) \leqq p<6\left[\right.$ Lemma 4.11(iv) and (P-4)] and $|x|^{-1} \in L^{4}$ $+L^{2}$ we have $g_{3} \in L^{\infty}$. Then the lemma follows from Eqs. (4.20a), (4.21), (4.24), (4.25) and Theorem 4.12.

We conclude with the proof that $\phi \in C^{2}\left(\mathbb{R}_{+}^{k}\lfloor\underline{0})\right.$ uniformly in $x$, with the following

Lemma 4.19. (Convergence of $\phi_{i j}^{\varepsilon}(\underline{z}, x)$ to $\phi_{i j}(\underline{z}, x)$ ): Let

$$
\phi_{i j}^{\varepsilon}(\underline{z}, x) \equiv \varepsilon^{-1}\left[\phi_{i}\left(\underline{z}+\varepsilon \underline{e}_{j}, x\right)-\phi_{i}(\underline{z}, x)\right]
$$

with $\underline{e}_{j}=\left[\delta_{j}^{j}\right]$ unit vector in $\mathbb{R}_{+}^{k}$ along $z_{j}$ and $\varepsilon \geqq-z_{j}$. Then $\phi_{i j}^{\varepsilon}(\underline{z}, x) \rightarrow \phi_{i j}(z, x)$ as $\varepsilon \rightarrow 0$, uniformly in $x$.

Proof. (i) Consider first $\varepsilon>0$. As in Lemma 4.14 we prove first:

$$
\phi_{i j}(\underline{z}, x) \leqq \phi_{i j}^{\varepsilon}(\underline{z}, x) \leqq \phi_{i j}\left(\underline{z}+\underline{e}_{j}, x\right) .
$$

Let $\psi(x)=\phi_{i j}^{\varepsilon}(z, x)-\phi_{i j}(\underline{z}, x)$. By Lemmas 4.11(i) and 4.15(i), $\psi$ is continuous everywhere and goes to zero at infinity. Then $S=\{x \mid \psi<0\}$ is open and $\psi=0$ on $\partial S \cup\{\infty\}$. Since $\varepsilon>0$, using Lemma 4.11(ii) we get,

$$
\begin{aligned}
& -(4 \pi)^{-1} \Delta \psi \geqq-(3 / 2) \phi(\underline{z}, x)^{1 / 2} \psi \\
& +(3 / 2 \varepsilon) \phi_{i}\left(\underline{z}+\varepsilon \underline{e}_{j}, x\right)\left[\phi(\underline{z}, x)^{1 / 2}-\phi\left(\underline{z}+\varepsilon \underline{e}_{j}, x\right)^{1 / 2}+(\varepsilon / 2) \phi(\underline{z}, x)^{-1 / 2} \phi_{j}(\underline{z}, x)\right] .
\end{aligned}
$$

Moreover, $\phi(\underline{z}, x)^{1 / 2}-\phi\left(\underline{z}+\varepsilon \underline{e}_{j}, x\right)^{1 / 2}+(1 / 2) \phi(\underline{z}, x)^{-1 / 2} \phi_{j}(z, x) \geqq 0$ because $\phi^{1 / 2}(\underline{z}, x)$ is concave (P-9), and $\phi$ is $C^{2}\left(\mathbb{R}_{+}^{k}\right)$ for each $x$. Therefore, on $S-(4 \pi)^{-1} \Delta \psi \geqq 0$ and by the MMP the first inequality in (4.26) follows. The other one is proved in the same way. Lemma 4.18 and (4.26) then imply $\left\|\phi_{i j}^{\varepsilon}(z, x)-\phi_{i j}(z, x)\right\|_{\infty} \rightarrow 0$ as $\varepsilon \downarrow 0$ for $\underline{z} \neq \underline{0}$.

(ii) If $-z_{j}<\varepsilon<0,(4.26)$ is replaced by $\phi_{i j}\left(\underline{z}+\varepsilon \underline{e}_{j}, x\right) \leqq \phi_{i j}^{\varepsilon}(\underline{z}, x) \leqq \phi_{i j}(\underline{z}, x)$ and the lemma follows from that.

\section{IV.3. Proof of Theorems 2.8-2.11: Properties of $K, A, R, e$, and $X$}

We begin by proving that $K(\underline{z})$ is in $C^{1}\left(\mathbb{R}_{+}^{k}\right)$ and $C^{2}\left(\mathbb{R}_{+}^{k} \backslash \underline{0}\right)$.

Lemma 4.20. (Existence of $\left.K_{i}(\underline{z})\right)$. Let $K(\underline{z}) \equiv(3 / 5) \int \phi(\underline{z}, x)^{5 / 2} d x$. Then

$$
K_{i}(\underline{z}) \equiv \lim _{\varepsilon \rightarrow 0} \varepsilon^{-1}\left[K\left(\underline{z}+\varepsilon \underline{e}_{i}\right)-K(\underline{z})\right]
$$

exists and is equal to $(3 / 2) \int \phi(\underline{z}, x)^{3 / 2} \phi_{i}(z, x) d x$, where $e_{i}=\left[\delta_{i}^{i}\right]$ is a unit vector along $z_{i}$.

Proof. (i) Consider first the case $\varepsilon \geqq 0$. Then

$$
\varepsilon^{-1}\left[K\left(\underline{z}+\varepsilon \underline{e}_{i}\right)-K(\underline{z})\right]=(3 / 5) \int \varepsilon^{-1}\left[\phi\left(\underline{z}+\varepsilon \underline{e}_{i}, x\right)^{5 / 2}-\phi(\underline{z}, x)^{5 / 2}\right] d x .
$$

Now,

$$
\varepsilon^{-1}\left[\phi\left(\underline{z}+\varepsilon \underline{e}_{i}, x\right)^{5 / 2}-\phi(\underline{z}, x)^{5 / 2}\right]=\varepsilon^{-1} P(\mu)\left[\phi\left(\underline{z}+\varepsilon \underline{e}_{i}, x\right)-\phi(\underline{z}, x)\right] \phi\left(\underline{z}+\varepsilon \underline{e}_{i}, x\right)^{3 / 2},
$$


where $P(\mu)=\left(1+\mu^{5 / 2}\right)^{-1}\left(1+\mu+\mu^{2}+\mu^{3}+\mu^{4}\right)$ and $\mu \equiv \phi(\underline{z}, x) \phi\left(\underline{z}+\varepsilon_{i}, x\right)^{-1}<1$ by (P-7). Hence $P(\mu) \leqq 5 / 2$. Moreover, because of (P-9) and Theorem 2.6,

$$
\phi\left(\underline{z}+\varepsilon \underline{e}_{i}, x\right)-\phi(\underline{z}, x) \leqq \varepsilon \phi_{i}(\underline{z}, x)
$$

Using (4.29), (4.27), and (4.28) we get:

$$
\begin{aligned}
\varepsilon^{-1}\left[\phi\left(\underline{z}+\varepsilon \underline{e}_{i}, x\right)^{5 / 2}-\phi(\underline{z}, x)^{5 / 2}\right] & \leqq(5 / 2) \phi_{i}(\underline{z}, x) \phi\left(\underline{z}+\varepsilon \underline{e}_{i}, x\right)^{3 / 2} \\
& \leqq(5 / 2)\left|x-R_{i}\right|^{-1} \phi\left(\underline{z}+\underline{e}_{i}, x\right)^{3 / 2}
\end{aligned}
$$

where the last inequality follows from (P-7) (assuming $\varepsilon \leqq 1$ ) and Lemma 4.11(ii). Since $\phi^{3 / 2}(\underline{z}, x) \in L^{1} \cap L^{2}$ at least, $\left|x-R_{i}\right|^{-1} \phi^{3 / 2}\left(\underline{z}+\underline{e}_{i}, x\right) \in L^{1}$. Hence the lemma follows by Theorem 2.6 and dominated convergence. (ii) In the case $-z_{i} \leqq \varepsilon \leqq 0$, an analysis similar to the above yields

$$
\begin{aligned}
\varepsilon^{-1}\left[\phi\left(\underline{z}+\varepsilon \underline{e}_{i}, x\right)^{5 / 2}-\phi(\underline{z}, x)^{5 / 2}\right] & \leqq 5 / 2 \phi_{i}\left(\underline{z}+\varepsilon \underline{e}_{i}, x\right) \phi(\underline{z}, x)^{3 / 2} \\
& \leqq(5 / 2)\left|x-R_{i}\right|^{-1} \phi(\underline{z}, x)^{3 / 2} \in L^{1}
\end{aligned}
$$

Lemma 4.20 assures us that the derivatives of $K(z)$ along the axis exist. The proof that $K(\underline{z})$ is in fact in $C^{1}\left(\mathbb{R}_{+}^{k}\right)$ is provided by the following:

Lemma 4.21. (Continuity of $\left.K_{i}(\underline{z})\right) . K_{i}(\underline{z}) \equiv(3 / 2) \int \phi(\underline{z}, x)^{3 / 2} \phi_{i}(\underline{z}, x) d x$ is continuous for all $\underline{\underline{z}} \in \mathbb{R}_{+}^{k}$.

Proof. Let us prove continuity at $\underline{z}$. Let $\underline{z}^{*}=2 \max _{1 \leqq i \leqq k}\left(z_{i}\right)(1,1, \ldots, 1)$. Let $A \equiv\left\{\underline{z}^{\prime} \in \mathbb{R}_{+}^{k} \mid \underline{z}^{*}-\underline{z}^{\prime} \in \mathbb{R}_{+}^{k}\right\}$. Obviously $\underline{z} \in A$. Consider $z^{\prime} \in A, \underline{z}^{\prime} \rightarrow \underline{z}$. By Corollary 2.7(iii),

$\phi\left(\underline{z}^{\prime}, x\right)^{3 / 2} \phi_{i}\left(\underline{z}^{\prime}, x\right) \leqq\left|x-R_{i}\right|^{-1} \phi\left(\underline{z}^{*}, x\right)^{3 / 2} \in L^{1}$.

Moreover, as $\underline{z}^{\prime} \rightarrow \underline{z}, \phi\left(\underline{z}^{\prime}, x\right)^{3 / 2} \phi_{i}\left(\underline{z}^{\prime}, x\right) \rightarrow \phi(\underline{z}, x)^{3 / 2} \phi_{i}(\underline{z}, x)$ everywhere (Theorem 2.6). The lemma follows by dominated convergence.

The following is a useful alternative expression for $K_{i}(z)$.

\section{Lemma 4.22.}

$$
K_{i}(\underline{z})=3 \lim _{x \rightarrow R_{i}}\left\{\phi(\underline{z}, x)-\sum_{j=1}^{k} z_{j} \phi_{j}(\underline{z}, x)\right\} .
$$

Note. Because of (P-3) and Theorem 2.6(iv), the above limit exists.

Proof. From Eqs. (4.1) and (4.2) we have

$$
\begin{aligned}
F(\underline{z}, x) & \equiv \phi(\underline{z}, x)-\sum_{j=1}^{k} z_{j} \phi_{j}(\underline{z}, x) \\
& =(3 / 2) \sum_{j=1}^{k} \int|x-y|^{-1} \phi(\underline{z}, y)^{1 / 2} z_{j} \phi_{j}(\underline{z}, y) d y-\int|x-y|^{-1} \phi(\underline{z}, y)^{3 / 2} d y .
\end{aligned}
$$


The two integrals on the right side of (4.31) are bounded and continuous everywhere, therefore,

$$
F\left(\underline{z}, R_{i}\right)=(3 / 2) \sum_{j=1}^{k} \int\left|y-R_{i}\right|^{-1} z_{j} \phi_{j}(z, y) \phi(\underline{z}, y)^{1 / 2} d y-\int\left|y-R_{i}\right|^{-1} \phi(z, y)^{3 / 2} d y .
$$

Because of Eq. (4.2) and Theorem 2.6(v) we have

$$
\int\left|y-R_{i}\right|^{-1} \phi(\underline{z}, y)^{1 / 2} \phi_{j}(\underline{z}, y) d y=\int\left|y-R_{j}\right|^{-1} \phi(\underline{z}, y)^{1 / 2} \phi_{i}(\underline{z}, y) d y
$$

for all $i, j$. From Eqs. (4.32) and (4.33) we get,

$$
F\left(\underline{z}, R_{i}\right)=(3 / 2) \int \phi(\underline{z}, y)^{1 / 2} \phi_{i}(z, y) \sum_{j=1}^{k} z_{j}\left|y-R_{j}\right|^{-1} d y-\int\left|y-R_{i}\right|^{-1} \phi(\underline{z}, y)^{3 / 2} d y .
$$

Combining the last equation and Eqs. (4.1) and (4.2) we finally get

$$
F\left(\underline{z}, R_{i}\right)=(1 / 2) \int \phi(\underline{z}, y)^{3 / 2} \phi_{i}(\underline{z}, y) d y=K_{i}(\underline{z}) / 3,
$$

which is Eq. (4.30). Note that to get the first equality we have used

$$
\begin{aligned}
& \int d y \phi(z, y)^{1 / 2} \phi_{i}(\underline{z}, y)\left[\int d w|w-y|^{-1} \phi(z, w)^{3 / 2}\right] \\
& \quad=\int d y \phi(\underline{z}, y)^{3 / 2}\left[\int d w|w-y|^{-1} \phi(z, w)^{1 / 2} \phi_{i}(\underline{z}, w)\right],
\end{aligned}
$$

which is true by Fubini's theorem, since $\phi(z, y)^{1 / 2} \phi_{i}(z, y) \in L^{1}$ (Lemma 4.10) and $\int d w|w-y|^{-1} \phi(\underline{z}, w)^{3 / 2} \in L^{\infty}$ (Theorem IV.1, [1]).

The right side of (4.30) can be written in terms of the right sides of the integral Eqs. (4.1) and (4.2). Using the same kind of dominated convergence argument as in the proof of Lemmas 4.20 and 4.21 , it is easy to check that $K_{i}$ is differentiable and

$$
\begin{aligned}
K_{i j}(z) & =-3 \sum_{l=1}^{k} z_{l} \phi_{l j}\left(z, R_{i}\right) \\
& =-3 \sum_{l=1}^{k} z_{l} \phi_{i j}\left(z, R_{l}\right),
\end{aligned}
$$

where the last equality follows by using Theorem 2.6.

Proof of Theorem 2.8. $K \in C^{1}, K \in C^{2}\left(\mathbb{R}^{k} \backslash \underline{0}\right)$ follow from Lemmas 4.20, 4.21, Eq. (4.34) and Theorem 2.6. (i) is proved in Lemma 4.22 and (ii) is Eq. (4.34).

Proof of Theorem 2.10. (i) Let us start with the convexity of $R(z)$. Define $z \equiv \alpha z_{1}$ $+(1-\alpha) \underline{z}_{2}, \alpha \in[0,1]$. Consider now the following identity:

$$
\begin{aligned}
& 2\left[R(\underline{z})-\alpha R\left(\underline{z}_{1}\right)-(1-\alpha) R\left(\underline{z}_{2}\right)\right] \\
& =2 \int d x d y\left[\phi(\underline{z}, x)^{3 / 2}-\alpha \phi\left(\underline{z}_{1}, x\right)^{3 / 2}-(1-\alpha) \phi\left(\underline{z}_{2}, x\right)^{3 / 2}\right]|x-y|^{-1} \phi(z, y)^{3 / 2} \\
& \quad-\alpha \int d x d y\left[\phi(\underline{z}, x)^{3 / 2}-\phi\left(\underline{z}_{1}, x\right)^{3 / 2}\right]|x-y|^{-1}\left[\phi(\underline{z}, y)^{3 / 2}-\phi\left(\underline{z}_{1}, y\right)^{3 / 2}\right] \\
& \quad-(1-\alpha) \int d x d y\left[\phi(\underline{z}, x)^{3 / 2}-\phi\left(\underline{z}_{2}, x\right)^{3 / 2}\right]|x-y|^{-1}\left[\phi(\underline{z}, y)^{3 / 2}-\phi\left(\underline{z}_{2}, y\right)^{3 / 2}\right] .
\end{aligned}
$$


The last two terms of the left side of (4.35) are negative because $|x|^{-1}$ is a positive kernel. Moreover from Eq. (4.1) we get the following identity

$$
\begin{gathered}
\int d x d y|x-y|^{-1}\left[\phi(z, x)^{3 / 2}-\alpha \phi\left(\underline{z}_{1}, x\right)^{3 / 2}-(1-\alpha) \phi\left(\underline{z}_{2}, x\right)^{3 / 2}\right] \phi(z, y)^{3 / 2} \\
=-\int d x d y\left[\phi(z, x)-\alpha \phi\left(\underline{z}_{1}, x\right)-(1-\alpha) \phi\left(\underline{z}_{2}, x\right)\right] \phi(z, y)^{3 / 2} \leqq 0,
\end{gathered}
$$

where the last inequality follows from the concavity of $\phi(z, x)(\mathrm{P}-9)$. From (4.35) and (4.36) the convexity follows. The SSA is proved in a similar way. The Virial theorem (Theorem II.23, [1]) yields

$$
A(\underline{z})=(5 / 3) K(\underline{z})+2 R(\underline{z}),
$$

and hence the convexity and SSA of $A$ follow from those of $K$ [Corollary 2.9(ii)] and $R$. (ii) That $e(z)$ is WSA on $\mathbb{R}_{+}^{k}$ is proven in [1], Theorem V.7. See also [3], Theorem 1. (This is in fact Teller's Theorem [5]).

Proof of Theorem 2.11. Equation (2.4) and Theorem 2.8 imply

$$
X_{i}(z)=2 K_{i}(\underline{z})-\sum_{j=1}^{k} z_{j} K_{j i}(\underline{z})
$$

Using now (2.2), (2.3), and (4.38) we get

$$
X_{i}(z)=\lim _{x \rightarrow R_{i}} 6 N(z, x),
$$

with

$$
N(\underline{z}, x)=\phi(\underline{z}, x)-\sum_{j=1}^{k} z_{j} \phi_{j}(\underline{z}, x)+\left(\frac{1}{2}\right) \sum_{j, l=1}^{k} z_{l} z_{j} \phi_{j l}(\underline{z}, x) .
$$

Note that in order to obtain (4.39) we have used $\phi \in C^{2}$ and also Theorem 2.6(v). Note also that the limit in (4.39) in fact exists because of (P-2), Theorem 2.6(iv) and (ii). Let us compute $N(\underline{z}+\underline{\varepsilon}, x)-N(\underline{z}, x)$ for $\underline{\varepsilon} \in \mathbb{R}_{+}^{k}$. Using (P-9) and $\phi \in C^{1}$ we have,

$$
\phi(\underline{z}+\underline{\varepsilon}, x)-\phi(\underline{z}, x) \geqq \sum_{j=1}^{k} \varepsilon_{j} \phi_{j}(\underline{z}+\underline{\varepsilon}, x) .
$$

From (4.40) and (4.41),

$$
\begin{aligned}
& N(\underline{z}+\underline{\varepsilon}, x)-N(\underline{z}, x) \geqq-\sum_{j=1}^{k} z_{j}\left[\phi_{j}(\underline{z}+\underline{\varepsilon}, x)-\phi_{j}(\underline{z}, x)\right] \\
& \quad+(1 / 2) \sum_{j, l=1}^{k}(\underline{z}+\underline{\varepsilon})_{l}(\underline{z}+\underline{\varepsilon})_{j} \phi_{j l}(\underline{z}+\underline{\varepsilon}, x)-(1 / 2) \sum_{l, j=1}^{k} z_{l} z_{j} \phi_{j l}(\underline{z}, x) .
\end{aligned}
$$

The ray-convexity of $\phi_{i}$ [Theorem 2.6(iii)] implies :

$$
\sum_{j=1}^{k} \varepsilon_{j} \phi_{i j}(\underline{z}, x) \leqq \phi_{i}(\underline{z}+\underline{\varepsilon}, x)-\phi_{i}(\underline{z}, x) \leqq \sum_{j=1}^{k} \varepsilon_{j} \phi_{i j}(\underline{z}+\underline{\varepsilon}, x) .
$$

We conclude that

$$
N(\underline{z}+\underline{\varepsilon}, x)-N(\underline{z}, x) \geqq(1 / 2) \sum_{j, l=1}^{k} \varepsilon_{l} \varepsilon_{j} \phi_{j l}(\underline{z}+\underline{\varepsilon}, x),
$$


since $\phi_{j l}(\underline{z}+\underline{\varepsilon}, x) \geqq \phi_{j l}(\underline{z}, x)$ [Lemma 4.15(iii)]. Although the right side of (4.43) is negative, it is second order in $\varepsilon$. The following Lemma 4.23 shows that under these conditions $N(z, x)$ must in fact be increasing. Hence $X_{i}(z)$ is increasing and by Lemma $2.1 X$ is SSA.

Lemma 4.23. Suppose $f:[a, b] \rightarrow \mathbb{R}$ is a real-valued function such that for every $x \in[a, b]$ there is a $c(x)$ such that $f(z)-f(x) \geqq c(x)(z-x)^{2}$ for all $z \in[a, b]$ with $z \geqq x$. Suppose further that $c \in L^{1}([a, b])$. Then $f$ is increasing, i.e. $z>x \Rightarrow f(z) \geqq f(x)$.

Proof. Let $N>1$ be an integer and let $I_{j}$, for $j=1, \ldots, n$ be the interval $I_{j}=(x+(j-1)(z-x) / n, x+j(z-x) / n)$. Then

$$
\delta \equiv f(z)-f(x)=\sum_{j=0}^{n}\left(f\left(y_{j+1}\right)-f\left(y_{j}\right)\right)
$$

with $y_{0}=x, y_{n+1}=z$ and $y_{j} \in I_{j}$. Without loss of generality we can assume $c(x) \leqq 0$, all $x$. Then

$$
\delta \geqq\left\{n^{-1} \sum_{j=0}^{n} c\left(y_{j}\right)\right\}\left\{4(z-x)^{2} / n\right\}
$$

because $y_{j+1}-y_{j} \leqq 2(z-x) / n$. Let $d_{j}=\int_{I_{j}} c(x) d x$ and $d=\sum_{j=1}^{n} d_{j}=\int_{x}^{z} c \geqq \int_{a}^{b} c>-\infty$. For each $j$, there exists $y_{j} \in I_{j}$ such that $c\left(y_{j}\right) \int_{I_{j}} 1 \geqq d_{j}$, otherwise $\int_{I_{J}} c<d_{j}$. Using these $y_{j}$ we have

$$
\delta \geqq\left\{n^{-1} c(x)+n^{-1} \sum_{j=1}^{n} d_{j}((z-x) / n)^{-1}\right\}\left\{4(z-x)^{2} / n\right\} .
$$

Taking $n \rightarrow \infty$ proves the lemma.

\section{Appendix A}

Properties of Superadditive and Convex Functions on $\mathbb{R}_{+}^{k}$

The definition of superadditive and convex functions on $\mathbb{R}_{+}^{k}$, as well as many of their properties, were stated in Section II. Those properties, Lemmas 2.1 to 2.4 and Corollary 2.5 , will be proved here.

Proof of Lemma 2.1. (i) $\Rightarrow$ is trivial because $f \in C^{2}\left(\mathbb{R}_{+}^{k}\right)$. To prove $\Leftarrow$, define $F(\lambda, \mu) \equiv f\left(\underline{z}+\lambda \underline{z}_{1}+\mu \underline{z}_{2}\right)-f\left(\underline{z}+\lambda \underline{z}_{1}\right)-f\left(\underline{z}+\mu \underline{z}_{2}\right)+f(\underline{z})$. Then, for $\underline{z}_{1}, \underline{z}_{2} \in \mathbb{R}_{+}^{k}$,

$$
F_{\lambda}(\lambda, \mu) \equiv(\partial F(\lambda, \mu) / \partial \lambda)=\sum_{i=1}^{k}\left(z_{1}\right)_{i}\left[f_{i}\left(\underline{z}+\lambda \underline{z}_{1}+\mu \underline{z}_{2}\right)-f_{i}\left(z+\lambda \underline{z}_{1}\right)\right]
$$

and

$$
F_{\lambda \mu}(\lambda, \mu)=\sum_{i, j=1}^{k}\left(z_{1}\right)_{i}\left(z_{2}\right)_{j} f_{i j}\left(\underline{z}+\lambda \underline{z}_{1}+\mu \underline{z}_{2}\right) \geqq 0,
$$


where the last inequality follows from $f_{i j} \geqq 0$ all $i, j$. But $F_{\lambda}(\lambda, 0) \geqq 0$, and hence $F_{\lambda}(\lambda, \mu) \geqq 0$. Also, $F(0, \mu)=0$, and hence $F(\lambda, \mu) \geqq 0$. (ii) $\Rightarrow$ follows immediately from the definition of an SSA function and the fact that $f \in C^{1}\left(\mathbb{R}_{+}^{k}\right)$. To prove $\Leftarrow$ note that if $f \in C^{1}\left(\mathbb{R}_{+}^{k}\right)$ and $f_{i}$ is increasing we get, from (A.1), $F_{\lambda}(\lambda, \mu) \geqq 0$. But $F(0, \mu)=0$ and therefore $F(\lambda, \mu) \geqq 0$.

Proof of Lemma 2.2. This is similar to the previous one, taking into account that $f_{i j} \geqq 0$ all $i, j, i \neq j$, and $\underline{z}_{1} \cdot \underline{z}_{2}=0$ imply

$$
\sum_{i, j=1}^{k}\left(z_{1}\right)_{i}\left(z_{2}\right)_{j} f_{i j} \geqq 0 .
$$

Proof of Lemma 2.3. If $f$ is SSA, taking $\underline{z}_{3}=\underline{z}_{2}$, in definition (iii) we have that $f\left((1 / 2) \underline{z}_{1}+(1 / 2)\left(\underline{z}_{1}+2 \underline{z}_{2}\right)\right) \leqq\left(f\left(\underline{z}_{1}\right) / 2\right)+\left(f\left(\underline{z}_{1}+2 \underline{z}_{2}\right) / 2\right)$.

Lemma 2.4 is a well known fact for differentiable convex functions. See [8], for example.

Proof of Corollary 2.5.

$$
\begin{aligned}
f\left(\underline{z}_{1}+\underline{z}_{2}\right)-f\left(\underline{z}_{1}\right) & =\int_{0}^{1} \frac{d}{d \lambda}\left(f\left(\underline{z}_{1}+\lambda \underline{z}_{2}\right)\right) d \lambda \\
& =\int_{0}^{1} \sum_{i=1}^{k}\left(z_{2}\right)_{i} f_{i}\left(\lambda \underline{z}_{2}+\underline{z}_{1}\right) d \lambda \leqq \sum_{i=1}^{k}\left(z_{2}\right)_{i} f_{i}\left(\underline{z}_{2}+\underline{z}_{1}\right)
\end{aligned}
$$

because $f_{i}$ is increasing, by Lemma 2.4 . The other inequality is proved in the same way.

\section{Appendix B}

Positivity of the Pressure Under Separation Relative to a Plane (in the Symmetric Case)

Consider $2 k$ nuclei with coordinates $R_{1}, \ldots, R_{k}$ and $R_{-1}, \ldots, R_{-k}$ and strictly positive charges $z_{1}, \ldots, z_{k}, z_{-1}, \ldots, z_{-k}$ satisfying (for $i=1, \ldots, k$ )

$$
\begin{aligned}
z_{i} & =z_{-i} \\
R_{i}^{1} & =R_{-i}^{1}, R_{i}^{2}=R_{-i}^{2},-R_{-i}^{3}=R_{i}^{3}>0 .
\end{aligned}
$$

Let $e(l)$ denote the TF energy for this molecule when $R_{i}^{3}$ is replaced by $R_{i}^{3}+l, i>0$ and by $R_{i}^{3}-l, i<0$. The electron charge $\lambda$ is immaterial but is fixed at some value $\lambda \leqq 2 \sum_{i=1}^{k} z_{i}$

Theorem B.1. The pressure is strictly positive, i.e.,

$$
e(l)<e(0) \text { for } l>0 \text {. }
$$

Proof. The proof consists of showing that if the charge distribution (electron and nuclear) is cut in two parts at the $x^{3}=0$ plane, and then pulled apart by a distance 
$2 l$, the energy is lowered. Let $\varrho(x)$ be the TF density when $l=0$. Define $\varrho_{l}(x)$ by

$$
\begin{aligned}
& \varrho_{l}(x)=\varrho(x-(0,0, l)), x^{3} \geqq l \\
& \varrho_{l}(x)=\varrho(x+(0,0, l)), x^{3} \leqq-l \\
& \varrho_{l}(x)=0,-l<x^{3}<l .
\end{aligned}
$$

Clearly $\int \varrho_{l}=\int \varrho=\lambda$, and we will use $\varrho_{l}$ as a trial density for the $l$ problem. We will show that $\mathscr{E}_{l}\left(\varrho_{l}\right)<\mathscr{E}(\varrho)=e(0)$, where $\mathscr{E}_{l}$ (resp. $\left.\mathscr{E}\right)$ is the energy functional for $l$ (resp. 0). Obviously, $K\left(\varrho_{l}\right)=K(\varrho)$.

Let $D \subset \mathbb{R}^{3}$ be the domain $\left\{\left(x^{1}, x^{2}, x^{3}\right) \mid x^{3} \geqq 0\right\}$. For any function $f: f: D \rightarrow C$, let $W_{l}(f)=\int_{D} \int_{D} d x d y \bar{f}(x) f(y) K_{l}(x, y)$,

$$
K_{l}(x, y)=\left\{\left(x^{1}-y^{1}\right)^{2}+\left(x^{2}-y^{2}\right)^{2}+\left(x^{3}+y^{3}+2 l\right)^{2}\right\}^{-1 / 2} .
$$

In other words, $W_{l}(f)$ is the Coulomb interaction energy between a charge distribution $f$, supported on the $x^{3} \geqq 0$ side of the $x y$ plane, and its (complex conjugate) reflection through the plane $x^{3}=-l$. It is easy to see that

$$
\mathscr{E}_{l}\left(\varrho_{l}\right)-\mathscr{E}(\varrho)=W_{l}(\mu)-W_{0}(\mu),
$$

where $\mu$ is the charge density for $x^{3} \geqq 0$ for the $l=0$ problem, namely for $x^{3} \geqq 0$

$$
\mu(x)=-\varrho(x)+\sum_{j=1}^{k} z_{j} \delta\left(x-R_{j}\right) .
$$

Since $\mu \neq 0$, and $W_{0}(\mu)=\lim _{l \downarrow 0} W_{l}(\mu)$, the following Lemma B.2 completes the proof.

Lemma B.2. (Reflection Positivity of the Coulomb Potential). Let $f$ be a non-null function with support in $D=\left\{x \mid x^{3} \geqq 0\right\}$ and with $f \in L^{1}(D)$. Then, for $l>0, W_{l}(f)>0$, and $W_{l}(f)$ is a finite, strictly decreasing function of $l$. Moreover, $W_{l}(f)$ is a log convex function of $l$, vanishing at $l=\infty$.

Proof. Using the well-known representation for $|x|^{-1}$, we have that

$$
\begin{aligned}
K_{l}(x, y)= & \left(2 \pi^{2}\right)^{-1} \int d^{3} p|p|^{-2} \exp \left\{i \left[p^{1}\left(x^{1}-y^{1}\right)+p^{2}\left(x^{2}-y^{2}\right)\right.\right. \\
& \left.\left.+p^{3}\left(x^{3}+y^{3}+2 l\right)\right]\right\} \\
= & (2 \pi)^{-1} \int d^{2} p|p|^{-1} \bar{g}_{p}(x) g_{p}(y) \exp (-2|p| l)
\end{aligned}
$$

with $g_{p}(x)=\exp \left[i p^{1} x^{1}+i p^{2} x^{2}-|p| x^{3}\right]$.

We have used the fact that

$$
\int_{-\infty}^{\infty} d p^{3}\left[\left(p^{3}\right)^{2}+a^{2}\right]^{-1} \exp \left[i p^{3}\left(x^{3}+y^{3}+2 l\right)\right]=(\pi / a) \exp \left[-a\left(x^{3}+y^{3}+2 l\right)\right]
$$

when $x^{3}+y^{3}+2 l>0$, as it is here. For $p \in \mathbb{R}^{2}$, let $h(p)=\int_{D} f(x) g_{p}(x) d^{3} x$. Since $f \in L^{1}(D),|h(p)| \leqq\|f\|_{1}$, and $h(p)$ is null if and only if $f$ is null. For $l>0$ Fubini's theorem yields

$$
W_{l}(f)=(2 \pi)^{-1} \int d^{2} p|p|^{-1}|h(p)|^{2} \exp (-2|p| l) .
$$

The representation (B.4) proves the lemma. 


\section{References}

1. Lieb, E. H., Simon, B.: Advan. Math. 23, 22-116 (1977)

2. Lieb, E. H., Simon, B.: Phys. Rev. Letters 31, 681-683 (1973)

Lieb, E. H.: Proc. Int. Congress of Math., Vancouver (1974)

Lieb, E. H.: Rev. Mod. Phys. 48, 553-569 (1976)

3. Benguria, R., Lieb, E. H.: Ann. Phys. (N.Y.) 110, 34-45 (1978)

4. Balàsz, N.: Phys. Rev. 156, 42-47 (1967)

5. Teller, E.: Rev. Mod. Phys. 34, 627-631 (1962)

6. Reed, M., Simon, B.: Methods of modern mathematical physics. Vol. II, Fourier analysis and selfadjointness. New York: Academic Press 1975

7. Hille, E.: Proc. Nat. Acad. Sci. 62, 7-10 (1969)

8. Rockafellar, R. T.: Convex analysis. Princeton: University Press 1970

9. Faris, W.: Duke Math. J. 43, 365-372 (1976)

10. Strichartz, R. S. J. Math. Mech. 16, 1031-1060 (1967)

11. Lieb, E. H., Simon, B.: Monotonicity of the electronic contribution to the Born-Oppenheimer energy. J. Phys. B (London) 11, L537-542 (1979)

12. Brezis, H., Lieb, E. H.: Long range atomic potentials in Thomas-Fermi theory. Commun. math. Phys. (submitted)

Communicated by J. Glimm

Received July 24, 1978

\section{Note Added in Proof}

In a recent related work [12], H. Brezis and E. H. Lieb have proved that the interaction among neutral atoms in Thomas-Fermi theory behaves, for large separation $l$, like $\mathrm{Cl}^{-7}$. 